\title{
Eigenvalue Asymptotics for the Shrödinger Operator with Steplike Magnetic Field and Slowly Decreasing Electric Potential
}

\author{
By
}

\author{
Shin-ichi SHIRAI*
}

\begin{abstract}
In this paper we consider the two-dimensional Schrödinger operator of the form:$$
H_{V}=-\frac{\partial^{2}}{\partial x_{1}^{2}}+\left(\frac{1}{i} \frac{\partial}{\partial x_{2}}-b\left(x_{1}\right)\right)^{2}+V\left(x_{1}, x_{2}\right),
$$

where the magnetic field $B\left(x_{1}\right)=\operatorname{rot}\left(0, b\left(x_{1}\right)\right)$ is monotone increasing and steplike, namely the limits $\lim _{x_{1} \rightarrow \pm \infty} B\left(x_{1}\right)=B_{ \pm}$exist with $0<B_{-}<B_{+}<\infty$, and $V$ is the slowly power-decaying electric potential. The spectrum $\sigma\left(H_{0}\right)$ of the unperturbed operator $H_{0}\left(=H_{V}\right.$ with $\left.V=0\right)$ has the band structure and $H_{V}$ has the discrete spectrum in the gaps of the essential spectrum $\sigma_{\text {ess }}\left(H_{V}\right)=\sigma\left(H_{0}\right)$. The aim of this paper is to study the asymptotic distribution of the eigenvalues near the edges of the spectral gaps. Using the min-max argument, we prove that the classical Weyl-type asymptotic formula is satisfied under suitable assumptions on $B$ and $V$.
\end{abstract}

\section{$\S 1 . \quad$ Introduction}

In this paper we investigate the asymptotic distribution of eigenvalues of the two-dimensional magnetic Schrödinger operator. We consider the operator acting in $L^{2}\left(\mathbf{R}^{2}\right)$ of the form:

$$
H_{V}=-\frac{\partial^{2}}{\partial x_{1}^{2}}+\left(\frac{1}{i} \frac{\partial}{\partial x_{2}}-b\left(x_{1}\right)\right)^{2}+V\left(x_{1}, x_{2}\right),
$$

Communicated by T. Kawai. Received Jannuary 11, 2002. Revised February 27, 2002. 2000 Mathematics Subject Classification(s): Primary 35P20; Secondary 35J10, 81Q10.

* Department of Mathematics, Graduate School of Science, Osaka University, Toyonaka, Osaka 560-0043, Japan.

e-mail: shirai@math.sci.osaka-u.ac.jp 
where $\left(0, b\left(x_{1}\right)\right)$ is the magnetic vector potential and $V$ is the electric scalar potential decaying at infinity.

We consider the case where the magnetic field $B=B(t)$ depends only on one variable. The relation between the magnetic vector potential and the magnetic field is given by

$$
b\left(x_{1}\right)=\int_{0}^{x_{1}} B(t) d t .
$$

When the vector potential $\left(0, b\left(x_{1}\right)\right)$ gives a constant magnetic field and the electric potential decays at infinity, the eigenvalue distribution around the essential spectrum (the Landau levels) has been investigated by several authors (see, [Rai1], [Rai2], [Tam]).

The purpose of the present paper is to obtain similar eigenvalue asymptotics in the spectral gaps of the operator (1.1) in the case of certain nonconstant, non-vanishing, bounded magnetic field (see the condition (B.1) below) and slowly power-decaying electric scalar potential (see the condition (V) below). The main strategy is the min-max argument as in [Col], [Tam]. The case where $V$ decays like $|x|^{-m}(m \geq 1)$ at infinity will be discussed in a future work.

In what follows, we denote $\frac{\partial}{\partial x_{1}}, \frac{\partial}{\partial x_{2}}$ by $\partial_{1}, \partial_{2}$, respectively, and we set $D_{1}=-i \partial_{1}, D_{2}=-i \partial_{2}$. Set $\langle z\rangle=\left(1+|z|^{2}\right)^{1 / 2}$ for $z \in \mathbf{R}^{n}$. For each open subset $\Omega$ of $\mathbf{R}^{2}$, we denote by $C_{0}^{\infty}(\Omega)$ the space of smooth functions with compact support in $\Omega$.

We introduce the conditions for the magnetic field $B$ :

(B.1) $B$ is a real-valued $C^{2}$-function on $\mathbf{R}$. Moreover, $B$ is monotone increasing and there exist positive numbers $B_{ \pm}>0$ such that $B_{-}<B_{+}$and $\lim _{x_{1} \rightarrow \pm \infty} B\left(x_{1}\right)=B_{ \pm}$, respectively.

(B.2) \pm In addition to (B.1), there exist positive constants $M, M^{\prime}$ and $C$ such that $\left|B\left(x_{1}\right)-B_{ \pm}\right| \leq C\left\langle x_{1}\right\rangle^{-M}$ and $\left|\partial_{1} B\left(x_{1}\right)\right| \leq C\left\langle x_{1}\right\rangle^{-M^{\prime}}$ hold as $x_{1} \rightarrow \infty$.

The Schrödinger operator with magnetic field satisfying (B.1) is also known as the Iwatsuka model (see [Iwa]). The various spectral properties of the Iwatsuka model have been investigated under weaker assumptions on $B$ by [Iwa], $[\mathrm{M}-\mathrm{P}],[\mathrm{E}-\mathrm{K}]$. In particular, under the condition (B.1), the unperturbed operator

$$
H_{0}=-\frac{\partial^{2}}{\partial x_{1}^{2}}+\left(\frac{1}{i} \frac{\partial}{\partial x_{2}}-b\left(x_{1}\right)\right)^{2}
$$


is essentially self-adjoint on $C_{0}^{\infty}\left(\mathbf{R}^{2}\right)$ and the spectrum of $H_{0}$ is purely absolutely continuous and has a band structure, i.e.,

$$
\sigma\left(H_{0}\right)=\bigcup_{n=1}^{\infty}\left[\Lambda_{n}^{-}, \Lambda_{n}^{+}\right]
$$

holds for $\Lambda_{n}^{ \pm}=(2 n-1) B_{ \pm}(n=1,2, \ldots)$. Here $\sigma(\cdot)$ denotes the spectrum of an operator.

Next, we introduce conditions for the electric scalar potential $V$ :

(V) $V$ is a real-valued $C^{2}$-function on $\mathbf{R}^{2}$ and there exist positive numbers $m, m^{\prime}$ and $C$ such that $0<m<1,2 m<m^{\prime}$,

$$
\begin{aligned}
|V(x)| & \leq C\langle x\rangle^{-m} \\
\left|\partial_{1} V(x)\right|+\left|\partial_{2} V(x)\right| & \leq C\langle x\rangle^{-m^{\prime}}
\end{aligned}
$$

for all $x \in \mathbf{R}^{2}$.

The condition $(\mathrm{V})$ implies that $V$ is a relatively compact perturbation with respect to $H_{0}$ and then $H_{V}$ is essentially self-adjoint on $C_{0}^{\infty}\left(\mathbf{R}^{2}\right)$ ([A-H-S $]$, [L-S]). Thus, one expects that $H_{V}$ has discrete spectra, i.e., isolated eigenvalues of finite multiplicity, in the spectral gaps of $H_{0}$ and they may accumulate to the tips of the gap.

For $\mu>0$ and $a_{0} \in \mathbf{R}$, we introduce the volume functions

$$
\nu_{ \pm}\left(\mu ; a_{0}\right)=\frac{1}{2 \pi} \operatorname{vol}\left\{\left(x_{1}, x_{2}\right) \in \mathbf{R}^{2} \mid \pm x_{1}>a_{0}, \pm V\left(x_{1}, x_{2}\right)>\mu\right\} .
$$

For a positive, decreasing function $f$, we introduce a condition by

(T) The homogeneity condition

$$
\lim _{\varepsilon \downarrow 0} \limsup _{\mu \downarrow 0} \mu^{2 / m}(f((1-\varepsilon) \mu)-f((1+\varepsilon) \mu))=0
$$

holds and there exist positive numbers $C, \mu_{0}$ such that

$$
f(\mu) \geq C \mu^{-\frac{2}{m}}
$$

holds for all $\mu \in\left(0, \mu_{0}\right)$.

For a self-adjoint operator $A$ acting in a Hilbert space, set

$$
N((a, b) \mid A)=\operatorname{dim}\left(\operatorname{Ran} E_{A}((a, b))\right),
$$


where $E_{A}(I)$ denotes the spectral projection of $A$ on an interval $I$.

In what follows, we shall devote ourselves to obtaining the eigenvalue distribution in a gap $\left(\Lambda_{n}^{+}, \Lambda_{n+1}^{-}\right)$when the gap is not empty, i.e.,

$$
\Lambda_{n}^{+}<\Lambda_{n+1}^{-}
$$

holds. For notational convenience, we put $\Lambda_{0}^{+}=-\infty$.

The main result of this paper is the following theorem.

Theorem 1.1. $\quad$ Let $n \geq 0$ be an integer which satisfies (1.3). Suppose that $\left(\mathrm{B} .2_{+}(\right.$resp. (B.2)- $)$and $(\mathrm{V})$ hold with

$$
M>m, \quad M^{\prime}>3 m .
$$

Moreover, suppose that $\nu_{+}\left(\mu ; a_{0}\right)$ (resp. $\left.\nu_{-}\left(\mu ; a_{0}\right)\right)$ satisfies $(\mathrm{T})$ for some $a_{0}$. Then we have

$$
\begin{aligned}
N\left(\left(\Lambda_{n}^{+}+\mu, M_{n}\right) \mid H_{V}\right) & =B_{+} \nu_{+}\left(\mu ; a_{0}\right)(1+o(1)) \\
\text { (resp. } N\left(\left(M_{n}, \Lambda_{n+1}^{-}-\mu\right) \mid H_{V}\right) & \left.=B_{-} \nu_{-}\left(\mu ; a_{0}\right)(1+o(1)) \quad \text { as } \quad \mu \downarrow 0\right),
\end{aligned}
$$

where we put $M_{n}=\left(\Lambda_{n}^{+}+\Lambda_{n+1}^{-}\right) / 2$ for each $n \geq 1$ and $M_{0}=-\infty$.

We remark that, as $\mu \downarrow 0$, the leading term of asymptotics of $\nu_{ \pm}\left(\mu ; a_{0}\right)$ does not depend on the choice of $a_{0}$. In fact, if we replace $a_{0}$ by $a_{1}(\mu)=a_{0}+o\left(\mu^{-\frac{1}{m}}\right)$ as $\mu \downarrow 0$, then we have

$$
\left|\nu_{+}\left(\mu ; a_{0}\right)-\nu_{+}\left(\mu ; a_{1}(\mu)\right)\right|=o\left(\nu_{+}\left(\mu ; a_{0}\right)\right)
$$

as $\mu \downarrow 0$, because of the conditions $(\mathrm{V})$ and $(\mathrm{T})$ for $\nu_{+}\left(\mu ; a_{0}\right)$. A similar assertion holds for $\nu_{-}\left(\mu ; a_{0}\right)$. Thus, in the sequel, we shall denote $\nu_{ \pm}\left(\mu ; a_{0}\right)$ by $\nu_{ \pm}(\mu)$ for simplicity.

We shall give a proof only for the asymptotics for $N\left(\left(\Lambda_{n}^{+}+\mu, M_{n}\right) \mid H_{V}\right)$, since we can obtain the result for $N\left(\left(M_{n}, \Lambda_{n+1}^{-}-\mu\right) \mid H_{V}\right)$ in a similar way.

The plan of the paper is as follows. In Section 2, we shall state some results preparatory to the succeeding sections. In Section 3, we shall introduce a tessellation of $\mathbf{R}^{2}$. In Section 4 , we shall introduce several quadratic forms and reduce the problem to the ones on each regions of the tessellation and, using the min-max argument, we shall give an upper estimate for the number of eigenvalues. In Section 5, we shall give a lower estimate and complete the proof of the main theorem, accepting Proposition 2.1 in Section 2 below. In Section 6, we shall give a proof of Proposition 2.1. 


\section{§2. Preliminaries}

In what follows, we use the symbol " $C$ " to denote various positive constants in the place where any confusions do not occur. Its value may change from line to line. In the place where we emphasize the dependence on some variable, we denote $C_{\varepsilon}, C(\alpha), \ldots$ and so on.

Let $q=q[u, v]$ be a closable, symmetric sesqui-linear form bounded from below with domain $D(q)$, and let $H_{q}$ be the self-adjoint operator associated with the form closure $\bar{q}$ ([R-S1], Theorem VIII.15, [R-S2], Theorem X.23). For simplicity, we denote $N\left((-\infty, \mu) \mid H_{q}\right)$ by $N_{q}(\mu)$ and denote the quadratic form $q[u, u]$ by $q[u]$. In the sequel, we shall identify a sesqui-linear form with the associated quadratic form.

Lemma 2.1. For $j=1,2$, let $\left(\mathcal{H}_{j}, q_{j}, D\left(q_{j}\right)\right)$ be a triplet of a Hilbert space $\mathcal{H}_{j}$, a quadratic form $q_{j}$ and its form domain $D\left(q_{j}\right)$, and let $J$ an isometric operator from $D\left(q_{1}\right)$ to $D\left(q_{2}\right)$ with respect to the norms of $\mathcal{H}_{1}$ and $\mathcal{H}_{2}$ respectively. Suppose that there exist positive constants $C_{1}$ and $C_{2}$ such that

$$
q_{1}[u] \geq C_{1} q_{2}[J u]-C_{2}\|u\|_{\mathcal{H}_{1}}^{2}
$$

holds for all $u \in D\left(q_{1}\right)$. Then we have, for any $\mu \in \mathbf{R}$,

$$
N_{q_{1}}(\mu) \leq N_{q_{2}}\left(\left(\mu+C_{2}\right) / C_{1}\right)
$$

Proof. This is an easy consequence of the variational principle (see, e.g., [R-S4], Theorem XIII.2).

In what follows, we denote the operator domain by $\operatorname{Dom}(\cdot)$.

Lemma 2.2. $\quad$ Let $D_{0}$ be a dense subspace in a separable Hilbert space. Assume that a linear operator $A$ is closable on $D_{0}$. Define a quadratic form a on $D(a)=D_{0}$ by $a[u]=\|A u\|^{2}$. Then we have:

(i) The form closure is given by $\bar{a}[u]=\|\bar{A} u\|^{2}$ with form domain $D(\bar{a})=$ $\operatorname{Dom}(\bar{A})$ and the self-adjoint operator associated with $\bar{a}$ coincides with $(\bar{A})^{*} \bar{A}$. Here $\bar{A}$ is the operator closure of $A$ on $D_{0}$.

(ii) Moreover, if $A$ is essentially self-adjoint on $D_{0}$, then the identity

$$
N_{a}\left(\mu^{2}\right)=N((-\mu, \mu) \mid \bar{A})
$$

holds for any $\mu>0$. 
Proof. Set $\tilde{a}[u]=\|\bar{A} u\|^{2}$ with domain $D(\tilde{a})=\operatorname{Dom}(\bar{A})$. Then it is easy to see that the closedness of the operator $\bar{A}$ implies the closedness of the form $\tilde{a}$ and that the fact that $D_{0}$ is an operator core for $\bar{A}$ implies that $\tilde{a}$ coincides with $\bar{a}$. The rest of assertion (i) is obvious.

The assertion (ii) follows since $N_{a}\left(\mu^{2}\right)=N\left(\left(-\infty, \mu^{2}\right) \mid(\bar{A})^{2}\right)=N((-\mu, \mu)$ $\mid \bar{A})$.

In the following, we denote by $Q\left(z_{0}, R\right)$ the square in $\mathbf{R}^{2}$ centered at $z_{0} \in$ $\mathbf{R}^{2}$, of size $R \times R$, with sides parallel to the coordinate axes.

For $B_{0}>0$, we introduce the operators

$$
\Pi_{1}\left(B_{0}\right)=D_{1}-\frac{B_{0}}{2} x_{2}, \quad \Pi_{2}\left(B_{0}\right)=D_{2}+\frac{B_{0}}{2} x_{1}
$$

and set

$$
H\left(B_{0}\right)=\Pi_{1}\left(B_{0}\right)^{2}+\Pi_{2}\left(B_{0}\right)^{2} .
$$

For each $z_{0} \in \mathbf{R}^{2}, R>0$ and $\lambda \in \mathbf{R}$, we define the quadratic form $q=$ $q\left(B_{0}, \lambda, Q\left(z_{0}, R\right)\right)$ on $D(q)=C_{0}^{\infty}\left(Q\left(z_{0}, R\right)\right)$ by

$$
q[u]=\left\|\left(H\left(B_{0}\right)+\lambda\right) u\right\|_{L^{2}\left(Q\left(z_{0}, R\right)\right)}^{2} .
$$

It is easy to see that $q$ is a closable form in $L^{2}\left(Q\left(z_{0}, R\right)\right)$ by Lemma 2.2 .

The next proposition shall play an important role in the following sections. (We shall give a proof in Section 6.)

Proposition 2.1. Let $q=q\left(B_{0}, \lambda, Q\left(z_{0}, R\right)\right)$ be the form as above. We have:

(i) For any $\mu>0$,

$$
N_{q}\left(\mu^{2}\right) \leq \frac{1}{2 \pi} B_{0} R^{2} \sharp\left\{n \in N||(2 n-1) B_{0}+\lambda \mid<\mu\right\}
$$

holds. Here $\sharp$ denotes the cardinal number of a set.

(ii) There exist constants $C>0, \delta_{1}>0$ such that, for any $\eta$ and $\delta$ with $0<\eta<R / 2,0<\delta<\delta_{1}$,

$$
N_{q}\left(\mu^{2}\right) \geq \frac{1}{2 \pi} B_{0}(R-\eta)^{2} \sharp\left\{n \in \mathbf{N}||(2 n-1) B_{0}+\lambda \mid<\mu_{\lambda \eta \delta}\right\}
$$

holds for any $\mu>0$, where $\mu_{\lambda \eta \delta}>0$ is a number defined by the relation

$$
\mu_{\lambda \eta \delta}^{2}=(1-\delta) \mu^{2}-C\left(\delta \lambda^{2}+\frac{1}{\eta^{4} \delta^{3}}\right) .
$$


Lemma 2.3. Set $X_{1}=D_{1}$ and $X_{2}=D_{2}-b\left(x_{1}\right)$. For $u \in C_{0}^{\infty}\left(\mathbf{R}^{2}\right)$, the estimate

$$
\sum_{j=1}^{2}\left\|X_{j}^{2} u\right\|^{2} \leq C\left(\left\|H_{0} u\right\|^{2}+\|u\|^{2}\right)
$$

holds for some constant $C>0$.

Proof. Set $A=X_{1}+i X_{2}$ and $A^{*}=X_{1}-i X_{2}$. Then we have

$$
\left\|X_{j}^{2} u\right\|^{2} \leq C\left\|\left(A \pm A^{*}\right)^{2} u\right\|^{2} \leq C \sum\left\|A^{\sharp_{1}} A^{\sharp_{2}} u\right\|^{2},
$$

where $A^{\sharp j}$ denotes $A$ or $A^{*}$.

By direct computations, we have, on $C_{0}^{\infty}\left(\mathbf{R}^{2}\right)$,

$$
\begin{aligned}
A A^{*} & =H_{0}+B, \quad A^{*} A=H_{0}-B, \\
{\left[A, A^{*}\right] } & =2 B, \quad[A, B]=-i B^{\prime}, \quad\left[A^{*}, B\right]=-i B^{\prime} .
\end{aligned}
$$

Then it follows from the above relations that

$$
\begin{aligned}
& \left\|A A^{*} u\right\|^{2} \leq C\left(\left\|H_{0} u\right\|^{2}+\|u\|^{2}\right), \\
& \left\|A^{*} A u\right\|^{2} \leq C\left(\left\|H_{0} u\right\|^{2}+\|u\|^{2}\right),
\end{aligned}
$$

and

$$
\begin{aligned}
\left\|A^{2} u\right\|^{2} & =\left(\left(A^{*} A\right)^{2} u, u\right)-2\left(B A^{*} A u, u\right)+2 i\left(B^{\prime} A u, u\right) \\
& =\left(\left(H_{0}-B\right)^{2} u, u\right)-2\left(B\left(H_{0}-B\right) u, u\right)+2 i\left(A u, B^{\prime} u\right) \\
& \leq C\left(\left\|H_{0} u\right\|^{2}+\|u\|^{2}\right) .
\end{aligned}
$$

We can estimate $\left\|\left(A^{*}\right)^{2} u\right\|^{2}$ in the same way. Hence the statement follows from $(2.4)-(2.7)$.

\section{$\S 3 . \quad$ Partition of $\mathbf{R}^{2}$}

In order to employ the mini-max arguments, we introduce a tessellation and an associated open covering of $\mathbf{R}^{2}$.

For small $\delta_{0}>0$, set

$$
\sigma_{1}=1 / m-2 \delta_{0}, \quad \sigma_{2}=1 / m+\delta_{0}, \quad \sigma_{3}=1+\delta_{0}^{2},
$$

where $m$ is the constant as in (V). Then we observe that, for sufficiently small $\delta_{0}>0$, the relation

$$
1<\sigma_{3}<\sigma_{1}<1 / m<\sigma_{2}
$$


holds. Also, we take and fix $\delta_{0}$ sufficiently small depending only on $m, m^{\prime}, M$, $M^{\prime}$ in the way which will be specified later (see, (4.12), (4.33), (4.35)).

Set

$$
\begin{aligned}
\Omega_{0} & =\left\{\left(x_{1}, x_{2}\right) \in \mathbf{R}^{2}|| x_{1}\left|<\mu^{-\sigma_{1}},\right| x_{2} \mid<\mu^{-\sigma_{2}}\right\}, \\
\Omega_{1 \pm} & =\left\{\left(x_{1}, x_{2}\right) \in \mathbf{R}^{2}\left| \pm x_{1}>\mu^{-\sigma_{1}},\right| x_{2} \mid<\mu^{-\sigma_{2}}\right\}, \\
\Omega_{2 \pm} & =\left\{\left(x_{1}, x_{2}\right) \in \mathbf{R}^{2} \mid \pm x_{2}>\mu^{-\sigma_{2}}\right\}
\end{aligned}
$$

and

$$
\begin{aligned}
\widetilde{\Omega}_{0} & =\left\{\left(x_{1}, x_{2}\right) \in \mathbf{R}^{2}|| x_{1}\left|<\mu^{-\sigma_{1}}+\mu^{-\sigma_{3}},\right| x_{2} \mid<\mu^{-\sigma_{2}}+\mu^{-\sigma_{3}}\right\}, \\
\widetilde{\Omega}_{1 \pm} & =\left\{\left(x_{1}, x_{2}\right) \in \mathbf{R}^{2}\left| \pm x_{1}>\mu^{-\sigma_{1}}-\mu^{-\sigma_{3}},\right| x_{2} \mid<\mu^{-\sigma_{2}}+\mu^{-\sigma_{3}}\right\}, \\
\widetilde{\Omega}_{2 \pm} & =\left\{\left(x_{1}, x_{2}\right) \in \mathbf{R}^{2} \mid \pm x_{2}>\mu^{-\sigma_{2}}-\mu^{-\sigma_{3}}\right\} .
\end{aligned}
$$

If we set $K=\{0,1+, 1-, 2+, 2-\}$, then $\left\{\widetilde{\Omega}_{k}\right\}_{k \in K}$ forms an open covering of $\mathbf{R}^{2}$. Let $\left\{\varphi_{k}\right\}_{k \in K}$ be a set of functions such that $\operatorname{supp} \varphi_{k} \subset \widetilde{\Omega}_{k}$ and $0 \leq \varphi_{k} \leq 1$ hold for each $k, \sum_{k \in K} \varphi_{k}^{2}=1$, and for each $k \in K$ and for each multi-index $\alpha$,

$$
\left\|\partial^{\alpha} \varphi_{k}\right\|_{\infty} \leq C_{\alpha} \mu^{\sigma_{3}|\alpha|}
$$

holds, where the constant $C_{\alpha}>0$ is independent of $\mu, k$ and $\|\cdot\|_{\infty}$ denotes the supremum norm on $\mathbf{R}^{2}$.

\section{§4. Upper bound for the quantity $N_{h}\left(d_{n}^{2}\right)$}

\section{$\S 4.1$. Quadratic forms}

Let $\Lambda_{n}^{+}, M_{n}$ be as in Section 1. For each $\mu>0$, set

$$
a_{n}(\mu)=\frac{\left(\Lambda_{n}^{+}+2 \mu\right)+M_{n}}{2}, \quad d_{n}=\left(M_{n}-\Lambda_{n}^{+}\right) / 2 .
$$

and define the form $h$ on $D(h)=C_{0}^{\infty}\left(\mathbf{R}^{2}\right)$ by

$$
h[u]=\left\|\left(H_{V}-a_{n}(\mu)\right) u\right\|^{2} .
$$

Lemma 4.1. For any $\mu>0$ sufficiently small, we have

$$
\left|N\left(\left(\Lambda_{n}^{+}+\mu, M_{n}\right) \mid H_{V}\right)-N_{h}\left(d_{n}^{2}\right)\right| \leq C_{n},
$$

where $C_{n}$ is independent of $\mu$. 
Proof. We first note that

$$
a_{n}(\mu)-d_{n}=\Lambda_{n}^{+}+\mu, \quad a_{n}(\mu)+d_{n}=M_{n}+\mu .
$$

Applying Lemma 2.2 (ii) with $A=H_{V}-a_{n}(\mu), D_{0}=C_{0}^{\infty}\left(\mathbf{R}^{2}\right)$, we have

$$
\begin{aligned}
N_{h}\left(d_{n}^{2}\right) & =N\left(\left(-d_{n}, d_{n}\right) \mid H_{V}-a_{n}(\mu)\right) \\
& =N\left(\left(\Lambda_{n}^{+}+\mu, M_{n}+\mu\right) \mid H_{V}\right) \\
& =N\left(\left(\Lambda_{n}^{+}+\mu, M_{n}\right) \mid H_{V}\right)+N\left(\left[M_{n}, M_{n}+\mu\right) \mid H_{V}\right),
\end{aligned}
$$

where we used (4.3) in the last equality. Then the assertion follows from the fact that the eigenvalues of $H_{V}$ do not accumulate near $M_{n}$.

Lemma 4.2. Let $\varepsilon_{0}>0$ be sufficiently small. Then, there exists a constant $C>0$, independent of $\varepsilon_{0}$, such that the estimate

$$
h[u] \geq \sum_{k \in K}\left(1-C \varepsilon_{0}\right)\left\|\left(H_{V}-a_{n}(\mu)\right) \varphi_{k} u\right\|^{2}-C\left(\varepsilon_{0}+\mu^{4 \sigma_{3}} / \varepsilon_{0}^{3}\right)\|u\|^{2}
$$

holds for all $\mu>0$ small enough and for any $u \in C_{0}^{\infty}\left(\mathbf{R}^{2}\right)$. Here $\left\{\varphi_{k}\right\}_{k \in K}$ is as in the previous section.

Proof. In the following, we shall frequently use the elementary inequality

$$
\|a+b\|^{2} \geq\left(1-\varepsilon_{0}\right)\|a\|^{2}-C \varepsilon_{0}^{-1}\|b\|^{2},
$$

where the constant $C>0$ is independent of $a, b$ and $\varepsilon_{0}$.

For $u \in C_{0}^{\infty}\left(\mathbf{R}^{2}\right)$ and for small $\varepsilon_{0}>0$, we have

$$
\begin{aligned}
h[u] & =\left\|\left(H_{V}-a_{n}(\mu)\right) u\right\|^{2} \\
& =\sum_{k \in K}\left\|\varphi_{k}\left(H_{V}-a_{n}(\mu)\right) u\right\|^{2} \\
& =\sum_{k \in K}\left\|\left(H_{V}-a_{n}(\mu)\right) \varphi_{k} u+\left[\varphi_{k}, H_{V}\right] u\right\|^{2} \\
& \geq \sum_{k \in K}\left(\left(1-\varepsilon_{0}\right)\left\|\left(H_{V}-a_{n}(\mu)\right) \varphi_{k} u\right\|^{2}-\frac{C}{\varepsilon_{0}}\left\|\left[\varphi_{k}, H_{V}\right] u\right\|^{2}\right) .
\end{aligned}
$$

Let $X_{j}(j=1,2)$ be as in Lemma 2.3. Using the relation $H_{V}=X_{1}^{2}+X_{2}^{2}+$ $V$, we have

$$
\begin{aligned}
\sum_{k \in K}\left\|\left[\varphi_{k}, H_{V}\right] u\right\|^{2} & =\sum_{k \in K}\left\|\sum_{j=1}^{2}\left(2 i\left(\partial_{j} \varphi_{k}\right)\left(X_{j} u\right)+\left(\partial_{j}^{2} \varphi_{k}\right) u\right)\right\|^{2} \\
& \leq C \sum_{k \in K} \sum_{j=1}^{2}\left(\left\|\left(\partial_{j} \varphi_{k}\right) X_{j} u\right\|^{2}+\left\|\left(\partial_{j}^{2} \varphi_{k}\right) u\right\|^{2}\right) .
\end{aligned}
$$


Since it follows that

$$
\begin{aligned}
\sum_{k \in K} & \left\|\left(\partial_{j} \varphi_{k}\right) X_{j} u\right\|^{2} \\
= & \sum_{k \in K}\left(\left(\partial_{j} \varphi_{k}\right)^{2} X_{j}^{2} u, u\right)+\sum_{k \in K}\left(\left[X_{j},\left(\partial_{j} \varphi_{k}\right)^{2}\right] X_{j} u, u\right) \\
= & \sum_{k \in K}\left(X_{j}^{2} u,\left(\partial_{j} \varphi_{k}\right)^{2} u\right)-2 i \sum_{k \in K}\left(\left(\partial_{j} \varphi_{k}\right) X_{j} u,\left(\partial_{j}^{2} \varphi_{k}\right) u\right) \\
= & \left(X_{j}^{2} u, \sum_{k \in K}\left(\partial_{j} \varphi_{k}\right)^{2} u\right)-2 i \sum_{k \in K}\left(\left(\partial_{j} \varphi_{k}\right) X_{j} u,\left(\partial_{j}^{2} \varphi_{k}\right) u\right) \\
\leq & \varepsilon_{0}^{2}\left\|X_{j}^{2} u\right\|^{2}+\frac{C}{\varepsilon_{0}^{2}}\left\|\sum_{k \in K}\left(\partial_{j} \varphi_{k}\right)^{2} u\right\|^{2}+\varepsilon^{\prime} \sum_{k \in K}\left\|\left(\partial_{j} \varphi_{k}\right) X_{j} u\right\|^{2} \\
& +C_{\varepsilon^{\prime}} \sum_{k \in K}\left\|\left(\partial_{j}^{2} \varphi_{k}\right) u\right\|^{2},
\end{aligned}
$$

holds for any small $\varepsilon^{\prime}>0$, we have

$$
\begin{aligned}
& \sum_{k \in K} \sum_{j=1}^{2}\left\|\left(\partial_{j} \varphi_{k}\right) X_{j} u\right\|^{2} \\
& \quad \leq C \sum_{j=1}^{2}\left(\varepsilon_{0}^{2}\left\|X_{j}^{2} u\right\|^{2}+\frac{1}{\varepsilon_{0}^{2}}\left\|\sum_{k \in K}\left(\partial_{j} \varphi_{k}\right)^{2} u\right\|^{2}+\sum_{k \in K}\left\|\left(\partial_{j}^{2} \varphi_{k}\right) u\right\|^{2}\right)
\end{aligned}
$$

It follows from Lemma 2.3 that

$$
\begin{aligned}
& \sum_{j=1}^{2}\left\|X_{j}^{2} u\right\|^{2} \\
& \leq C\left(\left\|H_{0} u\right\|^{2}+\|u\|^{2}\right) \\
& \leq C\left(\left\|\left(H_{V}-a_{n}(\mu)\right) u\right\|^{2}+\left(a_{n}(\mu)^{2}+\|V\|_{\infty}+1\right)\|u\|^{2}\right) \\
& \leq C\left(\left\|\left(H_{V}-a_{n}(\mu)\right) u\right\|^{2}+\|u\|^{2}\right)
\end{aligned}
$$

holds for some constant $C>0$, independent of $\mu>0$. Then, from (4.6)-(4.8), we have

$$
\begin{aligned}
& \sum_{k \in K}\left\|\left[\varphi_{k}, H_{V}\right] u\right\|^{2} \\
& \leq C\left(\varepsilon_{0}^{2}\left\|\left(H_{V}-a_{n}(\mu)\right) u\right\|^{2}+\varepsilon_{0}^{2}\|u\|^{2}\right) \\
&+\varepsilon_{0}^{-2} C \sum_{j=1}^{2}\left(\left\|\sum_{k \in K}\left(\partial_{j} \varphi_{k}\right)^{2} u\right\|^{2}+\sum_{k \in K}\left\|\left(\partial_{j}^{2} \varphi_{k}\right) u\right\|^{2}\right) .
\end{aligned}
$$


Since it follows from (4.5) and (4.9) that

$$
\begin{aligned}
h[u] \geq & \left(1-\varepsilon_{0}\right) \sum_{k \in K}\left\|\left(H_{V}-a_{n}(\mu)\right) \varphi_{k} u\right\|^{2} \\
& -C\left(\varepsilon_{0} h[u]+\varepsilon_{0}\|u\|^{2}+\varepsilon_{0}^{-3}\left(\left\|\sum_{k \in K}\left(\partial_{j} \varphi_{k}\right)^{2} u\right\|^{2}+\sum_{k \in K}\left\|\left(\partial_{j}^{2} \varphi_{k}\right) u\right\|^{2}\right)\right),
\end{aligned}
$$

we have by $(3.3)$

$$
\begin{aligned}
& \left(1+C \varepsilon_{0}\right) h[u] \\
& \quad \geq\left(1-\varepsilon_{0}\right) \sum_{k \in K}\left\|\left(H_{V}-a_{n}(\mu)\right) \varphi_{k} u\right\|^{2}-C\left(\varepsilon_{0}+\mu^{4 \sigma_{3}} / \varepsilon_{0}^{3}\right)\|u\|^{2},
\end{aligned}
$$

from which the lemma immediately follows.

For each $k \in K$, define the form $h_{k}$ on $D\left(h_{k}\right)=C_{0}^{\infty}\left(\widetilde{\Omega}_{k}\right)$ by

$$
h_{k}[v]=\left\|\left(H_{V}-a_{n}(\mu)\right) v\right\|^{2} \quad\left(v \in D\left(h_{k}\right)\right) .
$$

Lemma 4.3. There exists a constant $C>0$ such that

$$
N_{h}\left(d_{n}^{2}\right) \leq \sum_{k \in K} N_{h_{k}}\left(\left(1+C \mu^{\sigma_{3}}\right) d_{n}^{2}\right)
$$

holds for any small $\mu>0$.

Proof. If we set $\varepsilon_{0}=\mu^{\sigma_{3}}$, then it follows from Lemma 4.2 that, for any $u \in C_{0}^{\infty}\left(\mathbf{R}^{2}\right)$,

$$
\begin{aligned}
h[u] & \geq \sum_{k \in K}\left(\left(1-C \mu^{\sigma_{3}}\right)\left\|\left(H_{V}-a_{n}(\mu)\right) \varphi_{k} u\right\|^{2}-C \mu^{\sigma_{3}}\left\|\varphi_{k} u\right\|^{2}\right) \\
& =\sum_{k \in K}\left(\left(1-C \mu^{\sigma_{3}}\right) h_{k}\left[\varphi_{k} u\right]-C \mu^{\sigma_{3}}\left\|\varphi_{k} u\right\|^{2}\right) .
\end{aligned}
$$

Define the form $q_{1}$ on $D\left(q_{1}\right)=\oplus_{k \in K} C_{0}^{\infty}\left(\widetilde{\Omega}_{k}\right)$ by

$$
q_{1}\left[\oplus_{k \in K} v_{k}\right]=\sum_{k \in K} h_{k}\left[v_{k}\right],
$$

and the isometry $J_{1}$ from $C_{0}^{\infty}\left(\mathbf{R}^{2}\right)$ to $\oplus_{k \in K} C_{0}^{\infty}\left(\widetilde{\Omega}_{k}\right)$ by $J_{1} u=\oplus_{k \in K}\left(\varphi_{k} u\right)$. Applying Lemma 2.1 to the pair of triplets $\left(L^{2}\left(\mathbf{R}^{2}\right), h, D(h)\right)$ and $\left(\oplus_{k \in K} L^{2}\left(\widetilde{\Omega}_{k}\right)\right.$, $\left.q_{1}, D\left(q_{1}\right)\right)$, we deduce from (4.10) that $N_{h}\left(d_{n}^{2}\right) \leq N_{q_{1}}\left(\left(1+C \mu^{\sigma_{3}}\right) d_{n}^{2}\right)$. 


\section{§4.2. Estimate of the form $h_{2, \pm}$}

Lemma 4.4. For any $\mu>0$ small enough, we have

$$
N_{h_{2 \pm}}\left(\left(1+C \mu^{\sigma_{3}}\right) d_{n}^{2}\right)=0 .
$$

Proof. Let $\mu>0$ be sufficiently small and let $u \in D\left(h_{2 \pm}\right)$. Since $\left|x_{2}\right|>$ $\mu^{-\sigma_{2}}-\mu^{-\sigma_{3}} \geq C \mu^{-\sigma_{2}}$ on $\widetilde{\Omega}_{2 \pm}$ by (3.2), we see that

$$
\left|V\left(x_{1}, x_{2}\right)\right| \leq C\left\langle x_{2}\right\rangle^{-m} \leq C \mu^{m \sigma_{2}} .
$$

Then, by (4.4), we have, for $\varepsilon=\mu^{\sigma_{3}}$,

$$
\begin{aligned}
h_{2 \pm}[u] & =\left\|\left(H_{V}-a_{n}(\mu)\right) u\right\|^{2} \\
& \geq(1-\varepsilon)\left\|\left(H_{0}-a_{n}(\mu)\right) u\right\|^{2}-C \varepsilon^{-1}\|V u\|^{2} \\
& \geq(1-\varepsilon)\left\|\left(H_{0}-a_{n}(\mu)\right) u\right\|^{2}-C \varepsilon^{-1} \sup _{\widetilde{\Omega}_{2 \pm}}\left|V\left(x_{1}, x_{2}\right)\right|^{2}\|u\|^{2} .
\end{aligned}
$$

From (3.2) and (4.11), it follows that $1-\varepsilon=1-o(\mu)$ and

$$
\varepsilon^{-1} \sup _{\widetilde{\Omega}_{2 \pm}}\left|V\left(x_{1}, x_{2}\right)\right|^{2} \leq C \mu^{2 m \sigma_{2}-\sigma_{3}}=O\left(\mu^{1+\left(2 m-\delta_{0}\right) \delta_{0}}\right)=o(\mu)
$$

hold as $\mu \downarrow 0$ if the condition

$$
\delta_{0}<2 m
$$

is satisfied.

If we define the form $q_{2}$ on $D\left(q_{2}\right)=C_{0}^{\infty}\left(\mathbf{R}^{2}\right)$ by

$$
q_{2}[u]=\left\|\left(H_{0}-a_{n}(\mu)\right) u\right\|^{2},
$$

and denote by $J_{2}$ is the natural injection from $D\left(h_{2 \pm}\right)$ to $D\left(q_{2}\right)$, then we have

$$
h_{2 \pm}[u] \geq(1-o(\mu)) q_{2}\left[J_{2} u\right]-o(\mu)\|u\|^{2} \quad \text { as } \quad \mu \downarrow 0 .
$$

Hence it follows from Lemma 2.1 that

$$
\begin{aligned}
N_{h_{2 \pm}}\left(\left(1+C \mu^{\sigma_{3}}\right) d_{n}^{2}\right) & \leq N_{q_{2}}\left(\tilde{d}_{n}(\mu)^{2}\right) \\
& =N\left(\left(a_{n}(\mu)-\tilde{d}_{n}(\mu), a_{n}(\mu)+\tilde{d}_{n}(\mu)\right) \mid H_{0}\right),
\end{aligned}
$$

where $\tilde{d}_{n}(\mu)$ is given by the relation of the form $\tilde{d}_{n}(\mu)^{2}=\left(d_{n}^{2}+o(\mu)\right) /(1-o(\mu))$ as $\mu \downarrow 0$. 
On the other hand, since $a_{n}(\mu)+\tilde{d}_{n}(\mu)=M_{n}+\mu+o(\mu)$ and $a_{n}(\mu)-\tilde{d}_{n}(\mu)$ $=\Lambda_{n}^{+}+\mu+o(\mu)$ hold as $\mu \downarrow 0$, and $\left(\Lambda_{n}^{+}, \Lambda_{n+1}^{-}\right) \cap \sigma\left(H_{0}\right)=\emptyset$, we have

$$
\left(a_{n}(\mu)-\tilde{d_{n}}(\mu), a_{n}(\mu)+\tilde{d_{n}}(\mu)\right) \cap \sigma\left(H_{0}\right)=\emptyset .
$$

Then the result follows from (4.14) and (4.15).

\section{$\S 4.3$. Estimate of the form $h_{0}$}

For $i=\left(i_{1}, i_{2}\right) \in Z^{2}$, set

$$
Q(i)=\left\{\left(x_{1}, x_{2}\right) \in \mathbf{R}^{2} \mid i_{j}<x_{j}<i_{j}+1 \text { for } j=1,2\right\}
$$

and

$$
I=\left\{i \in Z^{2}|| i_{j} \mid \leq\left[\left[\mu^{-\sigma_{j}}+\mu^{-\sigma_{3}}\right]\right]+2 \text { for } j=1,2\right\},
$$

where $[[N]]$ expresses the largest integer less than or equal to $N$. Then it is easy to see that

$$
\widetilde{\Omega}_{0} \subset \bigcup_{i \in I} \overline{Q(i)}
$$

Here, - denotes the closure with respect to the usual topology of $\mathbf{R}^{2}$.

We denote the center of $Q(i)$ by $z_{i}$ and denote $Q\left(z_{i}, 1+\eta\right)$ by $Q_{\eta}(i)$ for $0<\eta<1$. Define the form $q_{3}$ on $D\left(q_{3}\right)=\bigoplus_{i \in I} C_{0}^{\infty}\left(Q_{\eta}(i)\right)$ by

$$
q_{3}\left[\oplus u_{i}\right]=\sum_{i \in I}\left\|\left(H_{0}-a_{n}(\mu)\right) u_{i}\right\|^{2} \quad\left(u_{i} \in C_{0}^{\infty}\left(Q_{\eta}(i)\right)\right) .
$$

Lemma 4.5. Let $q_{3}$ be as above and let $d_{n}$ be as in (4.1). Then, for any $C_{0}>0$, we have $N_{q_{3}}\left(C_{0} d_{n}^{2}\right)=o\left(\mu^{-2 / m}\right)$ holds as $\mu \downarrow 0$.

Proof. For each $i \in I$, we denote the center of $Q(i)$ by $z_{i}=\left(c_{i}, d_{i}\right)$ and set

$$
b_{i}\left(x_{1}\right)=\int_{0}^{c_{i}} B(t) d t+B\left(c_{i}\right)\left(x_{1}-c_{i}\right),
$$

and introduce the operator $K_{i}$ acting in $L^{2}\left(\mathbf{R}^{2}\right)$ by

$$
K_{i}=D_{1}^{2}+\left(D_{2}-b_{i}\right)^{2} .
$$

Then, for a fixed $\varepsilon_{1}>0$ sufficiently small,

$$
\left\|\left(H_{0}-a_{n}(\mu)\right) u\right\|^{2} \geq\left(1-\varepsilon_{1}\right)\left\|\left(K_{i}-a_{n}(\mu)\right) u\right\|^{2}-\frac{C}{\varepsilon_{1}}\left\|\left(H_{0}-K_{i}\right) u\right\|^{2}
$$


holds for any $u \in C_{0}^{\infty}\left(Q_{\eta}(i)\right)$.

We set

$$
\begin{aligned}
r_{i}\left(x_{1}\right) & =b\left(x_{1}\right)-b_{i}\left(x_{1}\right) \\
& =\int_{c_{i}}^{x_{1}}\left(B(t)-B\left(c_{i}\right)\right) d t .
\end{aligned}
$$

Then it follows from the relation

$$
H_{0}-K_{i}=-2 r_{i}\left(D_{2}-b_{i}\right)+r_{i}^{2}
$$

that, for any small $\varepsilon_{2}>0$,

$$
\begin{aligned}
& \left\|\left(H_{0}-K_{i}\right) u\right\|^{2} \\
& \leq C\left\{\sup _{Q_{\eta}(i)}\left|r_{i}\right|^{2}\left\|\left(D_{2}-b_{i}\right) u\right\|^{2}+\sup _{Q_{\eta}(i)}\left|r_{i}\right|^{4}\|u\|^{2}\right\} \\
& \leq C \varepsilon_{2} \sup _{Q_{\eta}(i)}\left|r_{i}\right|^{2}\left\|K_{i} u\right\|^{2}+C\left(\varepsilon_{2}^{-1} \sup _{Q_{\eta}(i)}\left|r_{i}\right|^{2}+\sup _{Q_{\eta}(i)}\left|r_{i}\right|^{4}\right)\|u\|^{2} \\
& \leq C \varepsilon_{2} \sup _{Q_{\eta}(i)}\left|r_{i}\right|^{2}\left\|\left(K_{i}-a_{n}(\mu)\right) u\right\|^{2} \\
& \quad+C^{\prime}\left(\varepsilon_{2}^{-1} \sup _{Q_{\eta}(i)}\left|r_{i}\right|^{2}+\sup _{Q_{\eta}(i)}\left|r_{i}\right|^{4}\right)\|u\|^{2},
\end{aligned}
$$

where we used the fact that

$$
\left\|\left(D_{2}-b_{i}\right) u\right\|^{2}=\left(\left(D_{2}-b_{i}\right)^{2} u, u\right) \leq\left(K_{i} u, u\right)
$$

in the second inequality and the fact that $a_{n}(\mu)$ is uniformly bounded in small $\mu>0$ in the third inequality.

Since the side length of $Q_{\eta}(i)$ is less than 2 units, we have

$$
\sup _{Q_{\eta}(i)}\left|r_{i}\right| \leq C\left\|B^{\prime}\right\|_{\infty}
$$

for some constant $C>0$. Then it follows from (4.16)-(4.18) that

$$
\begin{aligned}
\|\left(H_{0}-\right. & \left.a_{n}(\mu)\right) u \|^{2} \\
\geq & \left(1-\varepsilon_{1}\right)\left\|\left(K_{i}-a_{n}(\mu)\right) u\right\|^{2}-C \varepsilon_{2} \varepsilon_{1}^{-1}\left\|B^{\prime}\right\|_{\infty}^{2}\left\|\left(K_{i}-a_{n}(\mu)\right) u\right\|^{2} \\
& -C \varepsilon_{1}^{-1}\left(\varepsilon_{2}^{-1}\left\|B^{\prime}\right\|_{\infty}^{2}+\left\|B^{\prime}\right\|_{\infty}^{4}\right)\|u\|^{2} \\
\geq & \left(1-2 \varepsilon_{1}\right)\left\|\left(K_{i}-a_{n}(\mu)\right) u\right\|^{2}-C\left(\varepsilon_{1}^{-3}\left\|B^{\prime}\right\|_{\infty}^{2}+\varepsilon_{1}^{-1}\left\|B^{\prime}\right\|_{\infty}^{4}\right)\|u\|^{2},
\end{aligned}
$$

where we set $\varepsilon_{2}=\varepsilon_{1}^{2} / C$ in the second inequality. Then it is easy to see that

$$
q_{3}\left(\oplus_{i} u_{i}\right) \geq \sum_{i \in I}\left\{C\left\|\left(K_{i}-a_{n}(\mu)\right) u_{i}\right\|^{2}-C^{\prime}\left\|u_{i}\right\|^{2}\right\}
$$


for some positive constants $C, C^{\prime}>0$, independent of $\mu$ and $i$.

Finally it follows from (4.19), Lemma 2.1 and Proposition 2.1 that

$$
\begin{aligned}
& N_{q_{3}}\left(C_{0} d_{n}^{2}\right) \\
& \quad \leq \sum_{i \in I} \frac{1}{2 \pi} B\left(c_{i}\right)(1+\eta)^{2} \sharp\left\{n \in \mathbf{N}||(2 n-1) B\left(c_{i}\right)-\left.a_{n}(\mu)\right|^{2} \leq C d_{n}^{2}+C\right\} \\
& \quad \leq \sum_{i \in I} B\left(c_{i}\right) \frac{C}{B\left(c_{i}\right)} \\
& \quad \leq C \mu^{-\sigma_{1}-\sigma_{2}}=o\left(\mu^{-\frac{2}{m}}\right)
\end{aligned}
$$

as $\mu \downarrow 0$, where we used (3.2) in the last equality.

Lemma 4.6. $\quad$ Let $d_{n}$ be as in (4.1). For any $C>0$, we have

$$
N_{h_{0}}\left(\left(1+C \mu^{\sigma_{3}}\right) d_{n}^{2}\right)=o\left(\mu^{-2 / m}\right)
$$

as $\mu \downarrow 0$.

Proof. Let $\left\{\psi_{i}\right\}_{i \in Z^{2}}$ be a partition of unity of $\mathbf{R}^{2}$ such that $\sum_{i \in Z^{2}} \psi_{i}^{2}=1$, and, for each $i \in I, \psi_{i} \in C_{0}^{\infty}\left(Q_{\eta}(i)\right), 0 \leq \psi_{i} \leq 1, \psi_{i}(z)=\psi_{0}(z-i)$ hold and $\left|\partial^{\alpha} \psi_{i}\right| \leq C_{\alpha} \eta^{-|\alpha|}$ holds for each $\alpha \geq 0$.

We define the isometry $J_{3}$ from $D\left(h_{0}\right)=C_{0}^{\infty}\left(\widetilde{\Omega_{0}}\right)$ to $D\left(q_{3}\right)=$ $\oplus_{i \in I} C_{0}^{\infty}\left(Q_{\eta}(i)\right)$ by setting $J_{3} u=\oplus_{i \in I}\left(\psi_{i} u\right)$ for $u \in D\left(h_{0}\right)$. Since as in the proof of Lemma 4.2, we can show that, for a small $\varepsilon>0$,

$$
h_{0}[u] \geq(1-\varepsilon) q_{3}\left[\oplus_{i \in I}\left(\psi_{i} u\right)\right]-C\|u\|^{2}
$$

for some constant $C$ which depends only on $\varepsilon, \eta, V$ and $B$, applying Lemma 2.1 to $q_{3}, h_{0}$ and $J_{3}$, we have

$$
N_{h_{0}}\left(\left(1+C \mu^{\sigma_{3}}\right) d_{n}^{2}\right) \leq N_{q_{3}}\left(\frac{1+C}{1-\varepsilon} d_{n}^{2}\right) .
$$

Then the lemma obeys from Lemma 4.5.

\section{§4.4. Estimate of the form $h_{1-}$}

Lemma 4.7. Let $d_{n}$ be as in (4.1). Then, for any $C>0$, we have $N_{h_{1-}}\left(\left(1+C \mu^{\sigma_{3}}\right) d_{n}^{2}\right)=0$ for any $\mu>0$ sufficiently small. 
Proof. Since $x_{1} \leq-\mu^{\sigma_{1}}+\mu^{-\sigma_{3}} \leq-C \mu^{-\sigma_{1}}$ holds on $\widetilde{\Omega}_{1-}$, it follows from (B.1) and (V) that, as $\mu \downarrow 0$,

$$
\begin{aligned}
\left|V\left(x_{1}, x_{2}\right)\right| & \leq C\left\langle x_{1}\right\rangle^{-m} \leq C \mu^{m \sigma_{1}}, \\
B_{-} \leq B\left(x_{1}\right) & \leq B\left(-\mu^{\sigma_{1}}+\mu^{-\sigma_{3}}\right)=B_{-}+o(1) .
\end{aligned}
$$

By (4.4), we see that, as $\mu \downarrow 0$,

$$
\begin{aligned}
h_{1-}[u] & =\left\|\left(H_{V}-a_{n}(\mu)\right) u\right\|^{2} \\
& \geq\left(1-\mu^{m \sigma_{1}}\right)\left\|\left(H_{0}-a_{n}(\mu)\right) u\right\|^{2}-C \mu^{-m \sigma_{1}} \sup _{\widetilde{\Omega}_{1-}}|V|^{2}\|u\|^{2} \\
& \geq\left(1-\mu^{m \sigma_{1}}\right)\left\|\left(H_{0}-a_{n}(\mu)\right) u\right\|^{2}-O\left(\mu^{m \sigma_{1}}\right)\|u\|^{2} .
\end{aligned}
$$

Let $\beta$ be a number which satisfies

$$
B_{-}<\beta<B_{+}
$$

and let $\tilde{B}$ be a real-valued, monotonically increasing, smooth function on $\mathbf{R}$ which satisfies

$$
\tilde{B}\left(x_{1}\right)=\left\{\begin{aligned}
B\left(x_{1}\right), & \text { if } x_{1} \leq-\mu^{-\sigma_{1}}+\mu^{-\sigma_{3}}, \\
\beta, & \text { if } x_{1} \text { is sufficiently large, }
\end{aligned}\right.
$$

and the modified operator $\tilde{H}_{0}$ acting in $L^{2}\left(\mathbf{R}^{2}\right)$ by

$$
\tilde{H}_{0}=D_{1}^{2}+\left(D_{2}-\tilde{b}\right)^{2},
$$

where we set

$$
\tilde{b}\left(x_{1}\right)=\int_{0}^{x_{1}} \tilde{B}(t) d t+\int_{-\mu^{-\sigma_{1}+\mu^{-\sigma_{3}}}}^{0}(\tilde{B}(t)-B(t)) d t .
$$

Then we can find that

$$
H_{0} u=\tilde{H}_{0} u \quad \text { for } \quad u \in C_{0}^{\infty}\left(\widetilde{\Omega}_{1-}\right)
$$

and

$$
\sigma\left(\tilde{H}_{0}\right)=\bigcup_{n=1}^{\infty}\left[(2 k-1) B_{-},(2 k-1) \beta\right] .
$$

We denote by $q_{4}$ (resp. by $\left.q_{5}\right)$ the form $\left\|\left(\tilde{H}_{0}-a_{n}(\mu)\right) u\right\|^{2}$ on $C_{0}^{\infty}\left(\widetilde{\Omega}_{1-}\right)$ (resp. on $C_{0}^{\infty}\left(\mathbf{R}^{2}\right)$ ). Then we have, from (4.20) and (4.22),

$$
\begin{aligned}
& N_{h_{1-}}\left(\left(1+C \mu^{\sigma_{3}}\right) d_{n}^{2}\right) \\
& \left.\quad \leq N_{q_{4}}\left(\left(1+C \mu^{m \sigma_{1}}\right)\left(1+C \mu^{\sigma_{3}}\right) d_{n}^{2}\right)\right) \\
& \left.\quad \leq N_{q_{5}}\left(\left(1+C \mu^{m \sigma_{1}}\right)\left(1+C \mu^{\sigma_{3}}\right) d_{n}^{2}\right)\right) \\
& \quad \leq N\left(\left(a_{n}(\mu)-\left(1+C \mu^{m \sigma_{1}}\right) d_{n}, a_{n}(\mu)+\left(1+C \mu^{m \sigma_{1}}\right) d_{n}\right) \mid \tilde{H}_{0}\right),
\end{aligned}
$$


where we used Lemma 2.1 for the pair $h_{1-}$ and $q_{4}$ in the first inequality, and for the pair $q_{4}$ and $q_{5}$ in the second inequality.

On the other hand, since

$$
\begin{aligned}
& a_{n}(\mu)-\left(1+C \mu^{m \sigma_{1}}\right) d_{n}=\Lambda_{n}^{+}+O\left(\mu^{m \sigma_{1}}\right), \\
& a_{n}(\mu)+\left(1+C \mu^{m \sigma_{1}}\right) d_{n}=M_{n}+O\left(\mu^{m \sigma_{1}}\right)
\end{aligned}
$$

hold as $\mu \downarrow 0$, we deduce from (4.21), (4.23) that

(4.25) $\left(a_{n}(\mu)-\left(1+C \mu^{m \sigma_{1}}\right) d_{n}, a_{n}(\mu)+\left(1+C \mu^{m \sigma_{1}}\right) d_{n}\right) \cap \sigma\left(\tilde{H}_{0}\right)=\emptyset$

holds for any small $\mu>0$. The lemma follows from (4.24) and (4.25).

\section{§4.5. Estimate of the form $h_{1+}$}

For $i=\left(i_{1}, i_{2}\right) \in \boldsymbol{Z}^{2}$, set

$$
Q_{i}=\left\{\left(x_{1}, x_{2}\right) \in \mathbf{R}^{2} \mid i_{j}<\mu^{\sigma_{3}} x_{j}<i_{j}+1 \text { for } j=1,2\right\} .
$$

For $\eta$ with $0<\eta<1$, we denote the center of $Q_{i}$ by $z_{i}=\left(c_{i}, d_{i}\right)$ and we denote $Q_{i}\left(z_{i}, \mu^{-\sigma_{3}}(1+\eta)\right)$ by $Q_{i \eta}$.

Let $\left\{\psi_{i}\right\}_{i \in Z^{2}}$ be a partition of unity associated with the covering $\left\{Q_{i \eta}\right\}_{i \in Z^{2}}$ of $\mathbf{R}^{2}$ such that $\sum_{i \in Z^{2}} \psi_{i}^{2}=1$, and, for each $i \in Z^{2}, \psi_{i} \in C_{0}^{\infty}\left(Q_{i \eta}\right), 0 \leq \psi_{i} \leq 1$, $\psi_{i}\left(x_{1}, x_{2}\right)=\psi_{0}\left(x_{1}-i_{1} \mu^{-\sigma_{3}}, x_{2}-i_{2} \mu^{-\sigma_{3}}\right)$ and $\left|\partial^{\alpha} \psi_{i}\right| \leq C\left(\mu^{\sigma_{3}} / \eta\right)^{|\alpha|}$ holds for each $\alpha \geq 0$. If we set

$$
I^{\prime}=\left\{i \in Z^{2} \mid Q_{i \eta} \cap \widetilde{\Omega}_{1+} \neq \emptyset\right\},
$$

then $\sum_{i \in I^{\prime}} \psi_{i}^{2}=1$ holds on $\widetilde{\Omega}_{1+}$.

For each $i \in I^{\prime}$, we set

$$
\begin{aligned}
B_{i} & =B\left(c_{i}\right), \\
b_{i}\left(x_{1}\right) & =\int_{0}^{c_{i}} B(t) d t+B\left(c_{i}\right)\left(x_{1}-c_{i}\right), \\
V_{i}\left(x_{1}, x_{2}\right) & =V\left(z_{i}\right) \quad\left(=V\left(c_{i}, d_{i}\right)\right)
\end{aligned}
$$

and introduce the operator $H_{V, i}$ acting in $L^{2}\left(\mathbf{R}^{2}\right)$ by

$$
H_{V, i}=D_{1}^{2}+\left(D_{2}-b_{i}\right)^{2}+V_{i}
$$

Lemma 4.8. For any $u \in D\left(h_{1+}\right)$, the estimate

$$
h_{1+}[u] \geq \sum_{i \in I^{\prime}}\left(1-C \mu^{\sigma_{3}}\right)\left\|\left(H_{V, i}-a_{n}(\mu)\right) \psi_{i} u\right\|^{2}-\eta^{-4} o(\mu)\|u\|^{2}
$$

holds as $\mu \downarrow 0$ for some $C>0$. Here the term o( $\mu)$ is uniformly bounded with respect to small $\eta>0$. 
Proof. As in the proof of Lemma 4.2, we have, for $u \in C_{0}^{\infty}\left(\widetilde{\Omega}_{1+}\right)$ and for small $\varepsilon_{1}=\mu^{\sigma_{3}}>0$,

$$
\begin{aligned}
h_{1+}[u]= & \left\|\left(H_{V}-a_{n}(\mu)\right) u\right\|^{2} \\
\geq & \left(1-\varepsilon_{1}\right) \sum_{i \in I^{\prime}}\left\|\left(H_{V}-a_{n}(\mu)\right) \psi_{i} u\right\|^{2}-C \varepsilon_{1}\|u\|^{2} \\
& -C \frac{1}{\varepsilon_{1}^{3}} \sum_{j=1}^{2}\left(\left\|\sum_{k \in I^{\prime}}\left(\partial_{j} \psi_{k}\right)^{2} u\right\|^{2}+\sum_{k \in I^{\prime}}\left\|\left(\partial_{j}^{2} \psi_{k}\right) u\right\|^{2}\right) \\
\geq & \left(1-\varepsilon_{1}\right) \sum_{i \in I^{\prime}}\left\|\left(H_{V}-a_{n}(\mu)\right) \psi_{i} u\right\|^{2}-C \varepsilon_{1}\|u\|^{2} \\
& -C \mu^{4 \sigma_{3}} /\left(\eta^{4} \varepsilon_{1}^{3}\right)\|u\|^{2} \\
\geq & \left(1-\mu^{\sigma_{3}}\right) \sum_{i \in I^{\prime}}\left\|\left(H_{V}-a_{n}(\mu)\right) \psi_{i} u\right\|^{2}-C \eta^{-4} \mu^{\sigma_{3}}\|u\|^{2} .
\end{aligned}
$$

For each $i \in I^{\prime}$, set

$$
r_{i}=b\left(x_{1}\right)-b_{i}\left(x_{1}\right)=\int_{c_{i}}^{x_{1}}\left(B(t)-B\left(c_{i}\right)\right) d t .
$$

Then, since the condition $\left(x_{1}, x_{2}\right) \in Q_{\text {in }}$ implies that $x_{1} \geq C \mu^{-\sigma_{1}}$, using the mean value theorem, we have

$$
\sup _{Q_{i \eta}}\left|r_{i}\right| \leq \sup _{Q_{i \eta}} \sup _{t \geq C \mu^{-\sigma_{1}}}\left|B^{\prime}(t)\right|\left|x_{1}-c_{i}\right|^{2} \leq C \mu^{\sigma_{1} M^{\prime}-2 \sigma_{3}}
$$

and

$$
\begin{aligned}
\sup _{Q_{i \eta}}\left|V\left(x_{1}, x_{2}\right)-V_{i}\right| & \leq C \sup _{x_{1} \geq C \mu^{-\sigma_{1}}}\left|\nabla V\left(x_{1}, x_{2}\right)\right| \mu^{-\sigma_{3}} \\
& \leq C \mu^{\sigma_{1} m^{\prime}-\sigma_{3}}
\end{aligned}
$$

where we used the conditions (B.2) + and $(\mathrm{V})$.

From the relation

$$
H_{V}-H_{V, i}=-2 r_{i}\left(D_{2}-b_{i}\right)+r_{i}^{2}+\left(V-V_{i}\right),
$$

we have, for any $v \in C_{0}^{\infty}\left(Q_{i \eta}\right)$ and for any small $\varepsilon_{2}=\mu^{2 \sigma_{3}}>0$,

$$
\begin{aligned}
(4.31)\left\|\left(H_{V}-H_{V, i}\right) v\right\|^{2} & \\
& \leq C\left(\sup _{Q_{i \eta}}\left|r_{i}\right|^{2}\left\|\left(D_{2}-b_{i}\right) v\right\|^{2}+\sup _{Q_{i \eta}}\left|r_{i}\right|^{4}\|v\|^{2}+\sup _{Q_{i \eta}}\left|V-V_{i}\right|^{2}\|v\|^{2}\right) \\
& \leq C\left(\varepsilon_{2}\left\|\left(H_{V, i}-a_{n}(\mu)\right) v\right\|^{2}+\varepsilon_{2}^{-1} \sup _{Q_{i \eta}}\left|r_{i}\right|^{4}\|v\|^{2}+\sup _{Q_{i \eta}}\left|r_{i}\right|^{4}\|v\|^{2}\right.
\end{aligned}
$$




$$
\begin{aligned}
& \left.+\sup _{Q_{i \eta}}\left|V-V_{i}\right|^{2}\|v\|^{2}\right) \\
\leq & C \mu^{2 \sigma_{3}}\left\|\left(H_{V, i}-a_{n}(\mu)\right) v\right\|^{2} \\
& +C\left(\mu^{4 \sigma_{1} M^{\prime}-10 \sigma_{3}}+\mu^{4 \sigma_{1} M^{\prime}-8 \sigma_{3}}+\mu^{2 \sigma_{1} m^{\prime}-2 \sigma_{3}}\right)\|v\|^{2},
\end{aligned}
$$

where we used (4.29) and (4.30) in the third inequality. Then we have, for any $v \in C_{0}^{\infty}\left(Q_{i \eta}\right)$ and for small $\varepsilon_{3}=\mu^{\sigma_{3}}>0$,

$$
\begin{aligned}
\left\|\left(H_{V}-a_{n}(\mu)\right) v\right\|^{2} & \\
\geq & \left(1-\varepsilon_{3}\right)\left\|\left(H_{V, i}-a_{n}(\mu)\right) v\right\|^{2}-C \varepsilon_{3}^{-1}\left\|\left(H_{V}-H_{V, i}\right) v\right\|^{2} \\
\geq & \left(1-\varepsilon_{3}-C \varepsilon_{3}^{-1} \mu^{2 \sigma_{3}}\right)\left\|\left(H_{V, i}-a_{n}(\mu)\right) v\right\|^{2} \\
& \quad-C \varepsilon_{3}^{-1}\left(\mu^{4 \sigma_{1} M^{\prime}-10 \sigma_{3}}+\mu^{2 \sigma_{1} m^{\prime}-2 \sigma_{3}}\right)\|v\|^{2} \\
\geq & \left(1-C \mu^{\sigma_{3}}\right)\left\|\left(H_{V, i}-a_{n}(\mu)\right) v\right\|^{2}-C \mu^{\sigma_{4}}\|v\|^{2},
\end{aligned}
$$

where we used (4.31) in the second inequality, and we set

$$
\sigma_{4}=\min \left\{4 \sigma_{1} M^{\prime}-11 \sigma_{3}, 2 \sigma_{1} m^{\prime}-3 \sigma_{3}\right\}
$$

in the third inequality. Then since it follows from (1.4), (3.1) that

$$
\begin{gathered}
4 \sigma_{1} M^{\prime}-11 \sigma_{3}=4 \frac{M^{\prime}}{m}-11-\delta_{0}\left(8 M^{\prime}+11 \delta_{0}\right)>1, \\
2 \sigma_{1} m^{\prime}-3 \sigma_{3}=2 \frac{m^{\prime}}{m}-3-\delta_{0}\left(4 m^{\prime}+3 \delta_{0}\right)>1
\end{gathered}
$$

for sufficiently small $\delta_{0}>0$, the lemma follows from (4.28), (4.32).

Lemma 4.9. Let $d_{n}$ be as in (4.1). Then, for any $C>0$, we have

$$
\begin{aligned}
& N_{h_{1+}}\left(\left(1+C \mu^{\sigma_{3}}\right) d_{n}^{2}\right) \\
& \quad \leq \sum_{i \in I^{\prime}} \frac{B_{i}}{2 \pi} \mu^{-2 \sigma_{3}}(1+\eta)^{2} . \\
& \quad \sharp\left\{k \in N \|(2 k-1) B_{i}+V_{i}-a_{n}(\mu) \mid \leq\left(1+\eta^{-4} o(\mu)\right) d_{n}\right\}
\end{aligned}
$$

as $\mu \downarrow 0$, where $B_{i}=B\left(c_{i}\right), V_{i}=V\left(z_{i}\right)$. Here the term o( $\left.\mu\right)$ is uniformly bounded with respect to small $\eta>0$ and $i \in I^{\prime}$.

Proof. For each $i \in I^{\prime}$, define the form $q_{6, i}$ on $D\left(q_{6, i}\right)=C_{0}^{\infty}\left(Q_{i \eta}\right)$ by

$$
q_{6, i}[u]=\left\|\left(H_{V, i}-a_{n}(\mu)\right) v_{i}\right\|^{2} \quad\left(u \in D\left(q_{6, i}\right)\right)
$$


and define the form $q_{6}$ on $D\left(q_{6}\right)=\oplus_{i \in I^{\prime}} C_{0}^{\infty}\left(Q_{i \eta}\right)$ by

$$
q_{6}\left[\oplus_{i \in I^{\prime}}\left(v_{i}\right)\right]=\sum_{i \in I^{\prime}} q_{6, i}\left[v_{i}\right] \quad\left(v_{i} \in C_{0}^{\infty}\left(Q_{i \eta}\right)\right)
$$

and define the isometry $J_{6}$ from $D\left(h_{1+}\right)=C_{0}^{\infty}\left(\widetilde{\Omega}_{1+}\right)$ to $D\left(q_{6}\right)=\oplus_{i \in I^{\prime}} C_{0}^{\infty}\left(Q_{i \eta}\right)$ by $J_{6} u=\oplus_{i \in I^{\prime}}\left(\psi_{i} u\right)\left(u \in D\left(h_{1+}\right)\right)$. Then applying Lemma 2.1 to $h_{1+}, q_{6}$ and $J_{6}$, we have, by Lemma 4.8 ,

$$
\begin{aligned}
N_{h_{1+}} & \left(\left(1+C \mu^{\sigma_{3}}\right) d_{n}^{2}\right) \\
\leq & N_{q_{6}}\left(\frac{1+\eta^{-4} o(\mu)}{1-C \mu^{\sigma_{3}}}\left(1+C \mu^{\sigma_{3}}\right) d_{n}^{2}\right) \\
= & \sum_{i \in I^{\prime}} N_{q_{6, i}}\left(\frac{1+\eta^{-4} o(\mu)}{1-C \mu^{\sigma_{3}}}\left(1+C \mu^{\sigma_{3}}\right) d_{n}^{2}\right) \\
\leq & \sum_{i \in I^{\prime}} \frac{B_{i}}{2 \pi} \mu^{-2 \sigma_{3}}(1+\eta)^{2} . \\
& \sharp\left\{k \in N||(2 k-1) B_{i}+V_{i}-a_{n}(\mu) \mid<\left(1+\eta^{-4} o(\mu)\right) d_{n}\right\},
\end{aligned}
$$

where we used (4.34) and Proposition 2.1 in the third inequality.

To the end of this subsection, the quantities of the form $\left(1+\eta^{-4} o(\mu)\right) d_{n}$ are often abbreviated simply as $\tilde{d}(\mu ; \eta)$.

Lemma 4.10. Let $\tilde{d}(\mu ; \eta)$ be as above. Then

$$
\begin{gathered}
\sum_{i \in I^{\prime}} \frac{B_{i}}{2 \pi} \sharp\left\{k \in N||(2 k-1) B_{i}+V_{i}-a_{n}(\mu) \mid<\tilde{d}(\mu ; \eta)\right\} \\
\leq \sum_{i \in I^{\prime}} \frac{B_{i}}{2 \pi} F\left((2 n-1) B_{i}+V_{i}>a_{n}(\mu)-\tilde{d}(\mu ; \eta)\right)
\end{gathered}
$$

holds as $\mu \downarrow 0$, where $F(P)=1$ if a statement $P$ is true, $F(P)=0$ if $P$ is false.

Proof. We first note that the condition $\left|(2 k-1) B_{i}+V_{i}-a_{n}(\mu)\right|<\tilde{d}(\mu ; \eta)$ is equivalent to

$$
\begin{aligned}
(2 k-1) B_{i}+V_{i} & \in\left(a_{n}(\mu)-\tilde{d}(\mu ; \eta), a_{n}(\mu)+\tilde{d}(\mu ; \eta)\right) \\
& =\left(\Lambda_{n}^{+}+\mu-\eta^{-4} o(\mu), M_{n}+\mu+\eta^{-4} o(\mu)\right) .
\end{aligned}
$$

Since the conditions (B.1) and (V) implies the inequalities

$$
\begin{aligned}
\left|V_{i}\right| & \leq C \mu^{m \sigma_{1}}, \\
B_{+}-C \mu^{M \sigma_{1}} & \leq B\left(\mu^{-\sigma_{1}}-\mu^{-\sigma_{3}}\right) \leq B_{i} \leq B_{+},
\end{aligned}
$$


we can deduce that, for small $\mu \downarrow 0$,

$$
\begin{aligned}
& (2 k-1) B_{i}+V_{i} \leq(2 n-3) B_{i}+V_{i}<\Lambda_{n}^{+} \quad \text { if } k \leq n-1, \\
& (2 k-1) B_{i}+V_{i} \geq(2 n+1) B_{i}+V_{i} \geq \Lambda_{n+1}^{+}-o(1) \quad \text { if } k \geq n+1 .
\end{aligned}
$$

Then it follows that $\left|(2 k-1) B_{i}+V_{i}-a_{n}(\mu)\right|<\tilde{d}(\mu ; \eta)$ does not hold for any $k \geq n+1$, and $F\left((2 k-1) B_{i}+V_{i}>a_{n}(\mu)-\tilde{d}(\mu ; \eta)\right)=0$ holds for all $k \leq n-1$. Thus it is enough to consider only the case of $k=n$. It is easy to see that, for small $\mu>0$,

$$
\begin{aligned}
\sharp\{k & \left.\in \boldsymbol{N}||(2 k-1) B_{i}+V_{i}-a_{n}(\mu) \mid<\tilde{d}(\mu ; \eta)\right\} \\
& =\left\{\begin{array}{lll}
1, & \text { if } \quad(2 n-1) B_{i}+V_{i} \in\left(a_{n}(\mu)-\tilde{d}(\mu ; \eta),\right. & \left.a_{n}(\mu)+\tilde{d}(\mu ; \eta)\right), \\
0, & \text { if } \quad(2 n-1) B_{i}+V_{i} \notin\left(a_{n}(\mu)-\tilde{d}(\mu ; \eta),\right. & \left.a_{n}(\mu)+\tilde{d}(\mu ; \eta)\right),
\end{array}\right. \\
& \leq F\left((2 n-1) B_{i}+V_{i}>a_{n}(\mu)-\tilde{d}(\mu ; \eta)\right)
\end{aligned}
$$

This completes the proof.

For sufficiently small $\delta_{1}>0$, set

$$
F_{\delta_{1}}(\mu)=\left\{\left(x_{1}, x_{2}\right) \in \widetilde{\Omega}_{1+} \mid V\left(x_{1}, x_{2}\right)>\mu\left(1-\delta_{1}\right)\right\}
$$

and set

$$
\begin{aligned}
& I_{1}=\left\{i \in I^{\prime} \mid Q_{i} \subset F_{\delta_{1}}(\mu)\right\}, \\
& I_{2}=I^{\prime} \backslash I_{1} .
\end{aligned}
$$

Lemma 4.11. For small $\mu>0$,

$$
F\left((2 n-1) B_{i}+V_{i}>a_{n}(\mu)-\tilde{d}(\mu ; \eta)\right)=0
$$

holds uniformly in $i \in I_{2}$.

Proof. We choose $\delta_{0}>0$ sufficiently small so that

$$
\sigma_{1} m^{\prime}-\sigma_{3}>1, \quad \sigma_{1} M>1 .
$$

Then, since $\sup _{Q_{i \eta}}\left|V-V_{i}\right| \leq C \mu^{\sigma_{1} m^{\prime}-\sigma_{3}}=o(\mu)$ holds as $\mu \downarrow 0$, we have

$$
\begin{aligned}
\sup _{i \in I_{2}}\left((2 n-1) B_{i}+V_{i}\right) & \leq \Lambda_{n}^{+}+C \mu^{M \sigma_{1}}+\mu\left(1-\delta_{1}\right)+o(\mu) \\
& \leq \Lambda_{n}^{+}+\mu\left(1-\delta_{1}\right)+o(\mu) \\
& <a_{n}(\mu)-\tilde{d}(\mu ; \eta)
\end{aligned}
$$

as $\mu \downarrow 0$, where we used (B.2) + in the first inequality and used (1.4) in the second inequality. This proves the lemma. 
Lemma 4.12. Let $C>0$. Then we have

$$
\limsup _{\mu \downarrow 0} N_{h_{1+}}\left(\left(1+C \mu^{\sigma_{3}}\right) d_{n}^{2}\right) / B_{+} \nu_{+}(\mu) \leq 1 .
$$

Proof. It follows from Lemmas 4.9-4.11 that

$$
\begin{aligned}
N_{h_{1+}}\left(\left(1+C \mu^{\sigma_{3}}\right) d_{n}^{2}\right) & \leq \sum_{i \in I_{1}} \frac{B_{i}}{2 \pi} \mu^{-2 \sigma_{3}}(1+\eta)^{2} \\
& \leq \frac{B_{+}}{2 \pi} \mu^{-2 \sigma_{3}}(1+\eta)^{2}\left(\sharp I_{1}\right) \\
& \leq \frac{B_{+}}{2 \pi} \operatorname{vol} F_{\delta_{1}}(\mu)(1+\eta)^{2},
\end{aligned}
$$

where we used the fact that $\mu^{-2 \sigma_{3}}\left(\sharp I_{1}\right)=\operatorname{vol}\left(\bigcup_{i \in I_{1}} Q_{i}\right) \leq \operatorname{vol} F_{\delta_{1}}(\mu)$. Hence, taking Remark 1.1 into account, we have

$$
\begin{aligned}
\limsup _{\mu \downarrow 0} N_{h_{1+}}\left(\left(1+C \mu^{\sigma_{3}}\right) d_{n}^{2}\right) / B_{+} \nu_{+}(\mu) & \leq \limsup _{\mu \downarrow 0} \frac{1}{2 \pi} \frac{\operatorname{vol} F_{\delta_{1}}(\mu)}{\nu_{+}(\mu)}(1+\eta)^{2} \\
& \leq \underset{\mu \downarrow 0}{\limsup } \frac{\nu_{+}\left(\mu\left(1-\delta_{1}\right)\right)}{\nu_{+}(\mu)}(1+\eta)^{2} .
\end{aligned}
$$

Therefore, using the condition $(\mathrm{T})$ for $\nu_{+}$and the arbitrariness of the choice of $\eta, \delta_{1}$, we complete the proof.

Combining Lemmas 4.3, 4.4, 4.6, 4.7 and 4.12, we have:

\section{Proposition 4.1.}

$$
\limsup _{\mu \downarrow 0} N_{h}\left(d_{n}^{2}\right) / B_{+} \nu_{+}(\mu) \leq 1 .
$$

\section{$\S 5$. Lower Bound for the Quantity $N_{h}\left(d_{n}^{2}\right)$}

For each $i=\left(i_{1}, i_{2}\right) \in Z^{2}$, set

$$
Q_{i}=\left\{\left(x_{1}, x_{2}\right) \in \mathbf{R}^{2} \mid i_{j}<x_{j} \mu^{\sigma_{3}}<i_{j}+1 \text { for } j=1,2\right\}
$$

and set, for a fixed small number $\delta_{2}>0$,

$$
\begin{aligned}
F_{\delta_{2}}^{\prime}(\mu) & =\left\{\left(x_{1}, x_{2}\right) \in \mathbf{R}^{2} \mid x_{1}>\mu^{-\sigma_{1}}, V\left(x_{1}, x_{2}\right)>\mu\left(1+\delta_{2}\right)\right\}, \\
I_{0} & =\left\{i \in Z^{2} \mid Q_{i} \cap F_{\delta_{2}}^{\prime}(\mu) \neq \emptyset\right\}
\end{aligned}
$$


Let the operator $H_{V, i}$ be as in (4.27). For each $i \in I_{0}$, we introduce the form $q_{7, i}$ on $D\left(q_{7, i}\right)=C_{0}^{\infty}\left(Q_{i}\right)$ by

$$
q_{7, i}[u]=\left\|\left(H_{V, i}-a_{n}(\mu)\right) u\right\|^{2} \quad\left(u \in D\left(q_{7, i}\right)\right)
$$

and define the form $q_{7}$ on $D\left(q_{7}\right)=\oplus_{i \in I_{0}} C_{0}^{\infty}\left(Q_{i}\right)$ by

$$
q_{7}\left(\oplus_{i} u_{i}\right)=\sum_{i \in I_{0}} q_{7, i}\left[u_{i}\right] \quad\left(\oplus_{i \in I_{0}}\left(u_{i}\right) \in D\left(q_{7}\right)\right) .
$$

Lemma 5.1. Let $h$ be the form as in (4.2) and let $d_{n}$ be the number as in (4.1). Let $B_{i}, V_{i}$ and $b_{i}$ be as in (4.26). For any small $\eta>0$, we have

$$
\begin{aligned}
& N_{h}\left(d_{n}^{2}\right) \\
& \quad \geq \sum_{i \in I_{0}} \frac{B_{i}}{2 \pi}(1-\eta)^{2} \mu^{-2 \sigma_{3}} \sharp\left\{k \in N||(2 k-1) B_{i}+V_{i}-a_{n}(\mu) \mid<(1+o(\mu)) d_{n}\right\}
\end{aligned}
$$

as $\mu \downarrow 0$, where the term $o(\mu)$ is uniformly bounded with respect to $i \in I_{0}$.

Proof. Let $J_{7}$ be the natural isometry from $\oplus_{i \in I_{0}} C_{0}^{\infty}\left(Q_{i}\right)$ to $C_{0}^{\infty}\left(\mathbf{R}^{2}\right)$ defined by

$$
J_{7}\left(\oplus_{i} u_{i}\right)=\sum_{i \in I_{0}} u_{i} \quad\left(u_{i} \in C_{0}^{\infty}\left(Q_{i}\right)\right) .
$$

Then we have, as in the proof of Lemma 4.2,

$$
\begin{aligned}
h\left[J_{7}\right. & \left.\left(\oplus u_{i}\right)\right] \\
& =\sum_{i \in I_{0}}\left\|\left(H_{V}-a_{n}(\mu)\right) u_{i}\right\|^{2} \\
& =\sum_{i \in I_{0}}\left\|\left(H_{V, i}-a_{n}(\mu)\right) u_{i}+\left(H_{V}-H_{V, i}\right) u_{i}\right\|^{2} \\
& \leq \sum_{i \in I_{0}}\left(\left(1+\mu^{\sigma_{3}}\right)\left\|\left(H_{V, i}-a_{n}(\mu)\right) u_{i}\right\|^{2}+C / \mu^{\sigma_{3}}\left\|\left(H_{V}-H_{V, i}\right) u_{i}\right\|^{2}\right)
\end{aligned}
$$

and, as in the proof of Lemma 4.8, we obtain

$$
\begin{aligned}
\left\|\left(H_{V}-H_{V, i}\right) u_{i}\right\|^{2} & \\
\leq & \mu^{2 \sigma_{3}}\left\|\left(H_{V, i}-a_{n}(\mu)\right) u_{i}\right\|^{2} \\
& +C\left(\mu^{-2 \sigma_{3}} \sup _{Q_{i}}\left|r_{i}\right|^{4}+\sup _{Q_{i}}\left|r_{i}\right|^{4}+\sup _{Q_{i}}\left|V-V_{i}\right|^{2}\right)\left\|u_{i}\right\|^{2} \\
& \quad C \mu^{2 \sigma_{3}}\left\|\left(H_{V, i}-a_{n}(\mu)\right) u_{i}\right\|^{2}+C \mu^{\sigma_{4}+\sigma_{3}}\left\|u_{i}\right\|^{2},
\end{aligned}
$$


where $\sigma_{4}>1$ is the constant defined by (4.32).

Hence it follows from (5.1), (5.2) that

$$
\begin{aligned}
& h\left[J_{7}\left(\oplus u_{i}\right)\right] \\
& \quad \leq \sum_{i \in I_{0}}\left(\left(1+C \mu^{\sigma_{3}}\right)\left\|\left(H_{V, i}-a_{n}(\mu)\right) u_{i}\right\|^{2}+O\left(\mu^{\sigma_{4}}\right)\left\|u_{i}\right\|^{2}\right) \\
& \quad=\left(1+C \mu^{\sigma_{3}}\right) q_{7}\left[\oplus u_{i}\right]+O\left(\mu^{\sigma_{4}}\right) \sum_{i \in I_{0}}\left\|u_{i}\right\|^{2} .
\end{aligned}
$$

By using Lemma 2.1 and Proposition 2.1 (ii), we deduce from (5.3) that, as $\mu \downarrow 0$,

$$
\begin{aligned}
& N_{h}\left(d_{n}^{2}\right) \geq N_{q_{7}}\left((1+o(\mu)) d_{n}^{2}\right) \\
& \quad=\sum_{i \in I_{0}} N_{q_{7, i}}\left((1+o(\mu)) d_{n}^{2}\right) \\
& \quad \geq \sum_{i \in I_{0}} \frac{B_{i}}{2 \pi} \mu^{-2 \sigma_{3}}(1-\eta)^{2} \sharp\left\{k \in N||(2 k-1) B_{i}+V_{i}-a_{n}(\mu) \mid<d_{\eta}(\mu)\right\},
\end{aligned}
$$

where $\eta>0$ is a sufficiently small number and $d_{\eta}(\mu)$ is given by the relation

$$
\begin{aligned}
d_{\eta}(\mu)^{2} & =\left(1-\mu^{\sigma_{3}}\right)(1-o(\mu)) d_{n}^{2}-C\left(\mu^{\sigma_{3}}+\left(\eta \mu^{-\sigma_{3}}\right)^{-4} \mu^{-3 \sigma_{3}}\right) \\
& =(1-o(\mu)) d_{n}^{2}-C \mu^{\sigma_{3}}\left(1+\eta^{-4}\right) \\
& =(1+o(\mu)) d_{n}^{2}+o(\mu)=(1+o(\mu)) d_{n}^{2} .
\end{aligned}
$$

as $\mu \downarrow 0$. The lemma follows from this.

Proposition 5.1. $\quad$ Let $h$ be the form as in (4.2) and let $d_{n}$ be the number as in (4.1). Then we have

$$
\liminf _{\mu \downarrow 0} N_{h}\left(d_{n}^{2}\right) / B_{+} \nu_{+}(\mu) \geq 1 .
$$

Proof. We see that, as $\mu \downarrow 0$,

$$
\begin{aligned}
B_{+} \geq B_{i}>B\left(\mu^{-\sigma_{1}}\right) & =B_{+}-O\left(\mu^{\sigma_{1} M}\right)=B_{+}-o(\mu), \\
o(1) & >V_{i}>\mu\left(1+\delta_{2}\right)-o(\mu)
\end{aligned}
$$

uniformly in $i \in I_{0}$, where we used the condition (B.2)+ in the first expression, and used (V) and the mean value theorem in the second one. Then we have, as $\mu \downarrow 0$,

$$
\begin{aligned}
\Lambda_{n}^{+}+o(1) & >(2 n-1) B_{i}+V_{i} \\
& >(2 n-1)\left(B_{+}-o(\mu)\right)+\mu\left(1+\delta_{2}\right)-o(\mu) \\
& =\Lambda_{n}^{+}+\mu\left(1+\delta_{2}\right)+o(\mu)
\end{aligned}
$$


and

$$
a_{n}(\mu)+d_{\eta}(\mu)=M_{n}+\mu+o(\mu), \quad a_{n}(\mu)-d_{\eta}(\mu)=\Lambda_{n}^{+}+\mu+o(\mu) .
$$

Then we have from (5.4), (5.5) that, for any $\mu>0$ sufficiently small,

$$
a_{n}(\mu)-d_{\eta}(\mu)<(2 n-1) B_{i}+V_{i}<a_{n}(\mu)+d_{\eta}(\mu)
$$

holds as $\mu \downarrow 0$. From this, as in the proof of Lemma 4.10, we can deduce that

$$
\sharp\left\{k \in N||(2 k-1) B_{i}+V_{i}-a_{n}(\mu) \mid<d_{\eta}(\mu)\right\} \geq 1 .
$$

Then it follows from Lemma 5.1 that

$$
\begin{aligned}
N_{h}\left(d_{n}^{2}\right) & \geq \sum_{i \in I_{0}} \frac{B_{i}}{2 \pi}(1-\eta)^{2} \mu^{-2 \sigma_{3}} \\
& \geq \frac{1}{2 \pi}\left(B_{+}-C \mu^{\sigma_{1} M}\right)(1-\eta)^{2} \mu^{-2 \sigma_{3}}\left(\sharp I_{0}\right) \\
& \geq \frac{1}{2 \pi}\left(B_{+}-C \mu^{\sigma_{1} M}\right)(1-\eta)^{2} \operatorname{vol} F_{\delta_{2}}^{\prime}(\mu),
\end{aligned}
$$

where we used (5.6) in the first inequality and the fact that

$$
\mu^{-2 \sigma_{3}}\left(\sharp I_{0}\right)=\operatorname{vol}\left(\bigcup_{i \in I_{0}} Q_{i}\right) \geq \operatorname{vol} F_{\delta_{2}}^{\prime}(\mu)
$$

in the third inequality.

On the other hand, since

$$
\begin{aligned}
F_{\delta_{2}}^{\prime}(\mu)= & \left\{\left(x_{1}, x_{2}\right) \in \mathbf{R}^{2} \mid x_{1}>a_{0}, V\left(x_{1}, x_{2}\right)>\mu\left(1+\delta_{2}\right)\right\} \\
& \backslash\left\{\left(x_{1}, x_{2}\right) \in \mathbf{R}^{2} \mid a_{0}<x_{1}<\mu^{-\sigma_{1}}, V\left(x_{1}, x_{2}\right)>\mu\left(1+\delta_{2}\right)\right\},
\end{aligned}
$$

we obtain, as $\mu \downarrow 0$,

$$
\begin{aligned}
\frac{1}{2 \pi} \operatorname{vol} & F_{\delta_{2}}^{\prime}(\mu) \\
= & \nu_{+}\left(\mu\left(1+\delta_{2}\right)\right) \\
& -\frac{1}{2 \pi} \operatorname{vol}\left\{\left(x_{1}, x_{2}\right) \in \mathbf{R}^{2} \mid a_{0}<x_{1}<\mu^{-\sigma_{1}}, V\left(x_{1}, x_{2}\right)>\mu\left(1+\delta_{2}\right)\right\} \\
\geq & \nu_{+}\left(\mu\left(1+\delta_{2}\right)\right)-C \mu^{-\sigma_{1}-1 / m} \\
= & \nu_{+}\left(\mu\left(1+\delta_{2}\right)\right)+o\left(\mu^{-2 / m}\right) .
\end{aligned}
$$

Thus we have

$$
\begin{aligned}
\liminf _{\mu \downarrow 0} N_{h}\left(d_{n}^{2}\right) / B_{+} \nu_{+} & \geq(1-\eta) \liminf _{\mu \downarrow 0} \frac{B_{+}-C \mu^{\sigma_{1} M}}{B_{+}} \frac{1}{2 \pi} \frac{\operatorname{vol} F_{\delta_{2}}^{\prime}(\mu)}{\nu_{+}(\mu)} \\
& \geq(1-\eta) \liminf _{\mu \downarrow 0} \nu_{+}\left(\mu\left(1+\delta_{2}\right)\right) / \nu_{+}(\mu) .
\end{aligned}
$$


Therefore the lemma follows from the condition (T) for $\nu_{+}$and the arbitrariness of the choice of $\eta>0, \delta_{2}>0$.

Accepting Proposition 2.1, we now obtain Theorem 1.1 because of Lemma 4.1, Propositions 4.1 and 5.1.

\section{§6. Proof of Proposition 2.3}

In order to complete the proof of Theorem 1.1, we give a proof of Proposition 2.1 in this section.

For $B_{0}>0$, let $H\left(B_{0}\right)$ be the Schrödinger operator with the constant magnetic field $B_{0}$ defined by (2.3). For $R>0$ fixed, we introduce the function space $\mathcal{E}\left(B_{0}, R\right)$ by

$$
\mathcal{E}\left(B_{0}, R\right)=\left\{u \in C^{\infty}\left(\mathbf{R}^{2}\right) \mid S_{j} u=u \quad(j=1,2)\right\},
$$

where we set

$$
S_{1} u\left(x_{1}, x_{2}\right)=u\left(x_{1}+R, x_{2}\right) e^{i \frac{B_{0}}{2} R x_{2}}, \quad S_{2} u\left(x_{1}, x_{2}\right)=u\left(x_{1}, x_{2}+R\right) e^{-i \frac{B_{0}}{2} R x_{1}} .
$$

It is easy to see that $B_{0} R^{2} \in 2 \pi \boldsymbol{Z}$ if and only if $S_{1} S_{2}=S_{2} S_{1}$, and that $H\left(B_{0}\right)$, $\Pi_{j}\left(B_{0}\right)(j=1,2)$ leave $\mathcal{E}$ invariant.

In what follows we fix $z_{0} \in \mathbf{R}^{2}$ and denote $Q\left(z_{0}, R^{\prime}\right)$ simply by $Q_{R^{\prime}}$ for $R^{\prime}>0$. We denote by $H\left(B_{0}, R\right)$ the operator closure of $\left.H\left(B_{0}\right)\right|_{\mathcal{E}_{Q_{R}}}$, where we set

$$
\mathcal{E}_{Q_{R}}=\left\{\text { the restriction of } f \text { on } Q_{R} \mid f \in \mathcal{E}\left(B_{0}, R\right)\right\} \quad\left(\subset L^{2}\left(Q_{R}\right)\right) .
$$

Proposition 6.1. Assume $B_{0} R^{2}=2 \pi N_{0}$ holds for some integer $N_{0}$. Then $H\left(B_{0}, R\right)$ is a self-adjoint operator. Moreover $H\left(B_{0}, R\right)$ has eigenvalues $\left\{(2 k-1) B_{0}\right\}_{k \in N}$ and each of them has multiplicity $N_{0}$.

Proof. For the proof, see, e.g., [Col], Section 2.

For each $\lambda \in \mathbf{R}$, we define the quadratic form $q=q\left(B_{0}, \lambda, Q_{R}\right)$ on $D(q)=$ $C_{0}^{\infty}\left(Q_{R}\right)$ by

$$
q[u]=\left\|\left(H\left(B_{0}\right)+\lambda\right) u\right\|_{L^{2}\left(Q_{R}\right)}^{2} \quad(u \in D(q)),
$$

We prove Proposition 2.3 (i). Let $R>0$ and take $R_{1}>0$ large enough with $B_{0} R_{1}^{2} \in 2 \pi \boldsymbol{Z}$, and let $l \geq 0$ be the largest integer which satisfies $l R \leq R_{1}$. 
We divide a square $Q^{\prime}=Q\left(z_{0}, l R\right)$ to mutually disjoint $l^{2}$ squares of size $R \times R$, and denote them by $\left\{Q_{j}^{\prime}\right\}_{j=1}^{l^{2}}$.

Define the form $q_{8}$ on $D\left(q_{8}\right)=\bigoplus_{j=1}^{l^{2}} C_{0}^{\infty}\left(Q_{j}^{\prime}\right)$ by

$$
q_{8}\left[\oplus_{j=1}^{l^{2}} f_{j}\right]=\sum_{j=1}^{l^{2}} q\left[f_{j}\right] \quad\left(f_{j} \in C_{0}^{\infty}\left(Q_{j}^{\prime}\right)\right),
$$

where the form $q$ is as in (6.3), and define the form $q_{9}$ on $D\left(q_{9}\right)=\left.\mathcal{E}\left(B_{0}, R_{1}\right)\right|_{Q_{R_{1}}}$ by

$$
q_{9}[f]=\left\|\left(H\left(B_{0}\right)+\lambda\right) f\right\|_{L^{2}\left(Q_{R_{1}}\right)}^{2} \quad\left(f \in D\left(q_{9}\right)\right) .
$$

Then it follows from Lemma 2.1 and the definition of $Q_{j}^{\prime}$ 's that

$$
N_{q_{8}}\left(\mu^{2}\right)=l^{2} N_{q}\left(\mu^{2}\right)
$$

If we define the isometry $J_{8}$ by

$$
J_{8}:\left.D\left(q_{8}\right) \ni \oplus_{j=1}^{l^{2}} f_{j} \mapsto \sum_{j=1}^{l^{2}} f_{j} \in \mathcal{E}\left(B_{0}, R_{1}\right)\right|_{Q_{R_{1}}},
$$

then we have $q_{8}[f]=q_{9}\left[J_{6} f\right]$ for all $f \in D\left(q_{8}\right)$. Then, applying Lemma 2.1, we deduce from (6.4) that

$$
l^{2} N_{q}\left(\mu^{2}\right) \leq N_{q_{9}}\left(\mu^{2}\right)
$$

Lemma 2.2 implies that the self-adjoint operator associated with $q_{9}$ is $\left(H\left(B_{0}, R_{1}\right)+\lambda\right)^{2}$, so it follows from Proposition 6.1 that

$$
N_{q_{9}}\left(\mu^{2}\right)=\frac{B_{0} R_{1}^{2}}{2 \pi} \sharp\left\{k \in \boldsymbol{N}||(2 k-1) B_{0}+\lambda \mid<\mu\right\} .
$$

Then, from (6.4)-(6.6), we have

$$
N_{q}\left(\mu^{2}\right) \leq \frac{B_{0} R_{1}^{2}}{2 \pi l^{2}} \sharp\left\{k \in N||(2 k-1) B_{0}+\lambda \mid<\mu\right\} .
$$

Since, by the definition of $l$, we have

$$
\frac{B_{0} R_{1}^{2}}{2 \pi l^{2}} \longrightarrow \frac{R^{2} B_{0}}{2 \pi} \quad \text { as } \quad R_{1} \rightarrow \infty,
$$

the lemma follows from (6.7). Then we complete the proof of Proposition 2.1 (i). 
In the rest of this section, we devote ourselves to proving Proposition 2.1 (ii). For any $\eta>0$ fixed, choose $R>0$ sufficiently large so that $0<\eta<R / 2$. For a large integer $l>0$, choose $R_{2}>0$ as the largest number such that $B_{0} R_{2}^{2} \in 2 \pi \boldsymbol{Z}$ and $R_{2} \leq l(R-\eta)$ hold. By the definition of $R_{2}$, we observe that

$$
\frac{B_{0}}{2 \pi} l^{2}(R-\eta)^{2}-1 \leq \frac{B_{0}}{2 \pi} R_{2}^{2},
$$

then, for any given $\varepsilon>0$ and for sufficiently large $l$,

$$
\begin{aligned}
R_{2} & >\left\{l^{2}(R-\eta)^{2}-2 \pi / B_{0}\right\}^{1 / 2} \\
& \geq l(R-\eta)\left(1-\frac{2 \pi C}{B_{0} l^{2}(R-\eta)^{2}}\right) \\
& \geq(l-\varepsilon)(R-\eta) .
\end{aligned}
$$

Thus we have, for any given $\varepsilon>0$ and for sufficiently large $l$,

$$
l(R-\eta) \geq R_{2}>(l-\varepsilon)(R-\eta) .
$$

Let $z_{0} \in \mathbf{R}^{2}$ fixed and we consider a partition of a square $Q\left(z_{0}, l(R-\eta)\right)$ into mutually disjoint $l^{2}$ squares $Q_{j}^{\prime}=Q\left(z_{j}, R-\eta\right)\left(j=1,2, \ldots, l^{2}\right)$, where $z_{j}$ is the center of $Q_{j}^{\prime}$. Note that the condition (6.8) implies that $\overline{Q\left(z_{0}, R_{2}\right)} \subset$ $\cup_{j=1}^{l^{2}} \overline{Q_{j}^{\prime}}$.

Let $\Omega$ be a subset of $\mathbf{R}^{2}$. For each $n=\left(n_{1}, n_{2}\right) \in Z^{2}$ and for any $R>0$, we denote by $\Omega+n R$ the set $\left\{\left(z_{1}+n_{1} R, z_{2}+n_{2} R\right) \in \mathbf{R}^{2} \mid z=\left(z_{1}, z_{2}\right) \in \Omega\right\}$.

Lemma 6.1. Let $\eta, R, R_{2}$ and $z_{j}$ be as above and set $Q_{j}=Q\left(z_{j}, R\right)$ $\left(j=1, \ldots, l^{2}\right)$. Then there exists a partition of unity of $\mathbf{R}^{2},\left\{\psi_{j}^{\sharp}\right\}_{j=1}^{l^{2}}$, such that $0 \leq \psi_{j}^{\sharp}(z) \leq 1$,

$$
\sum_{j=1}^{l^{2}} \psi_{j}^{\sharp}(z)^{2}=1 \quad \text { on } \quad \mathbf{R}^{2}, \quad \operatorname{supp} \psi_{j}^{\sharp} \subset \bigcup_{n \in Z^{2}}\left(Q_{j}+n R_{2}\right)
$$

for $j=1, \ldots, l^{2}$ and, for each multi-index $\alpha$,

$$
\left|\partial_{z}^{\alpha} \psi_{j}^{\sharp}(z)\right| \leq C_{\alpha} \eta^{-|\alpha|}
$$

hold, where the constant $C_{\alpha}$ is independent of $z, \eta$. Moreover, $\psi_{j}^{\sharp}\left(x_{1}, x_{2}\right)$ is periodic with periods $\left(R_{2}, 0\right),\left(0, R_{2}\right)$.

Proof. Let $\theta(z)$ be a smooth function on $Q(0, R)$ with compact support such that $0 \leq \theta(z) \leq 1, \theta(z)=1$ on $Q(0, R-\eta)$, and $\left|\partial^{\alpha} \theta(z)\right| \leq C_{\alpha} \eta^{-|\alpha|}$ holds 
for each multi-index $\alpha=\left(\alpha_{1}, \alpha_{2}\right)$. If we set $\theta_{j}(z)=\theta\left(z-z_{j}\right)$ for $z \in \mathbf{R}^{2}$, then $\theta_{j} \in C_{0}^{\infty}\left(Q_{j}\right)$ and $\theta_{j}=1$ holds on $\overline{Q_{j}^{\prime}}$.

Define

$$
\theta_{j}^{\sharp}(z)=\sum_{n \in Z^{2}} \theta_{j}\left(z-n R_{2}\right),
$$

then we have

$$
\theta_{j}^{\sharp}=1 \quad \text { on } \bigcup_{n \in Z^{2}}\left(\overline{Q_{j}^{\prime}}+n R_{2}\right), \quad \operatorname{supp} \theta_{j}^{\sharp} \subset \bigcup_{n \in Z^{2}}\left(Q_{j}+n R_{2}\right) .
$$

It follows from (6.8) for sufficiently large $l$ that $\left(Q_{j}+n R_{2}\right) \cap\left(Q_{j}+n^{\prime} R_{2}\right)=\emptyset$ if $n \neq n^{\prime}$, and, for each $k=1, \ldots, l^{2}$,

$$
\sharp\left\{j \mid \bigcup_{n \in Z^{2}}\left(Q_{j}+n R_{2}\right) \cap \bigcup_{n^{\prime} \in Z^{2}}\left(Q_{k}+n^{\prime} R_{2}\right) \neq \emptyset\right\} \leq 9 .
$$

If we set $\Theta=\sum_{j=1}^{l^{2}}\left(\theta_{j}^{\sharp}\right)^{2}$, then we can deduce from (6.11) and (6.12) that $1 \leq \Theta \leq 9$ holds.

Finally, we obtain the desired functions $\psi_{j}^{\sharp}=\theta_{j}^{\sharp} / \sqrt{\Theta}$ for $j=1, \ldots, l^{2}$.

We introduce the forms

$$
\begin{aligned}
q_{10}[u] & =\left\|\left(H\left(B_{0}\right)+\lambda\right) u\right\|_{L^{2}\left(Q_{R_{2}}\right)}^{2}, \\
D\left(q_{10}\right) & =\left.\mathcal{E}\left(B_{0}, R_{2}\right)\right|_{Q_{R_{2}}} \quad\left(\subset L^{2}\left(Q_{R_{2}}\right)\right)
\end{aligned}
$$

and

$$
\begin{aligned}
q_{11}\left[\oplus_{k=1}^{l^{2}} f_{k}\right] & =\sum_{k=1}^{l^{2}}\left\|\left(H\left(B_{0}\right)+\lambda\right) f_{k}\right\|_{L^{2}\left(Q_{k}\right)}^{2}, \\
D\left(q_{11}\right) & =\bigoplus_{k=1}^{l^{2}} C_{0}^{\infty}\left(Q_{k}\right) \quad\left(\subset \bigoplus_{k=1}^{l^{2}} L^{2}\left(Q_{k}\right)\right),
\end{aligned}
$$

and define a map $J_{10}$ by

$$
J_{10}:\left.D\left(q_{10}\right) \ni u \mapsto \oplus_{k=1}^{l^{2}}\left(\psi_{k}^{\sharp} \cdot u^{\sharp}\right)\right|_{Q_{k}} \in D\left(q_{11}\right),
$$

where $u^{\sharp}$ is the element of $\mathcal{E}\left(B_{0}, R_{2}\right)$ such that $\left.u^{\sharp}\right|_{Q_{R_{2}}}=u$.

Lemma 6.2. The map $J_{10}$ is an isometry from $D\left(q_{10}\right)$ to $D\left(q_{11}\right)$ with respect to norms of $L^{2}\left(Q_{R_{2}}\right)$ and $\bigoplus_{k=1}^{l^{2}} L^{2}\left(Q_{k}\right)$, respectively. 
Proof. By the definitions, we have

$$
\begin{aligned}
\left\|J_{10} u\right\|_{\oplus L^{2}\left(Q_{k}\right)}^{2} & =\sum_{k=1}^{l^{2}}\left\|\psi_{k}^{\sharp} u^{\sharp}\right\|_{L^{2}\left(Q_{k}\right)}^{2} \\
& =\sum_{k=1}^{l^{2}} \int_{Q_{k}} \psi_{k}^{\sharp}(z)^{2}\left|u^{\sharp}(z)\right|^{2} d z,
\end{aligned}
$$

and

$$
\begin{aligned}
\int_{Q_{k}} \psi_{k}^{\sharp}(z)^{2}\left|u^{\sharp}(z)\right|^{2} d z \\
\quad=\sum_{n \in Z^{2}} \int_{Q_{k} \cap\left(Q_{R_{2}}+n R_{2}\right)} \psi_{k}^{\sharp}(z)^{2}\left|u^{\sharp}(z)\right|^{2} d z \\
\quad=\sum_{n \in Z^{2}} \int_{\left(Q_{k}-n R_{2}\right) \cap Q_{R_{2}}} \psi_{k}^{\sharp}\left(z^{\prime}+n R_{2}\right)^{2}\left|u^{\sharp}\left(z^{\prime}+n R_{2}\right)\right|^{2} d z^{\prime} \\
=\int_{Q_{R_{2}}} \psi_{k}^{\sharp}(z)^{2}\left|u^{\sharp}(z)\right|^{2} d z,
\end{aligned}
$$

since it follows from Lemma 6.1 that $\psi_{k}^{\sharp}(z)^{2}\left|u^{\sharp}(z)\right|^{2}$ is a function with periods $\left(R_{2}, 0\right),\left(0, R_{2}\right)$ and $\operatorname{supp} \psi_{k}^{\sharp} \subset \bigcup_{n \in Z^{2}}\left(Q_{k}-n R_{2}\right)$. Hence it follows from (6.13) and (6.14) that

$$
\begin{aligned}
\left\|J_{10} u\right\|_{\oplus L^{2}\left(Q_{k}\right)}^{2} & =\sum_{k=1}^{l^{2}} \int_{Q_{R_{2}}} \psi_{k}^{\sharp}(z)^{2}\left|u^{\sharp}(z)\right|^{2} d z \\
& =\int_{Q_{R_{2}}}\left|u^{\sharp}(z)\right|^{2} d z \\
& =\|u\|_{L^{2}\left(Q_{R_{2}}\right)}^{2},
\end{aligned}
$$

where we used (6.9) in the second inequality. This shows that $J_{10}$ is an isometry.

Lemma 6.3. There exist $C>0$ and $\delta_{1}>0$ such that

$$
q_{10}[u] \geq(1-2 \delta) q_{11}\left[J_{10} u\right]-C\left(\delta \lambda^{2}+\frac{1}{\delta^{3} \eta^{4}}+\frac{1}{\delta \eta^{4}}\right)\|u\|_{L^{2}\left(Q_{R_{2}}\right)}^{2}
$$

holds for any positive numbers $\eta<R / 2$ and $\delta<\delta_{1}$ and for all $u \in D\left(q_{1}\right)$. 
Proof. By the same method as in the preceding sections, we have, for small $\delta>0$,

$$
\begin{aligned}
q_{10}[u]= & \left\|\left(H\left(B_{0}\right)+\lambda\right) u\right\|_{L^{2}\left(Q_{R_{2}}\right)}^{2} \\
= & \left.\| J_{10}\left(H\left(B_{0}\right)+\lambda\right) u\right) \|_{\oplus L^{2}\left(Q_{k}\right)}^{2} \\
= & \sum_{k=1}^{l^{2}}\left\|\psi_{k}^{\sharp}\left(H\left(B_{0}\right)+\lambda\right) u^{\sharp}\right\|_{L^{2}\left(Q_{k}\right)}^{2} \\
\geq & (1-\delta) \sum_{k=1}^{l^{2}}\left\|\left(H\left(B_{0}\right)+\lambda\right) \psi_{k}^{\sharp} u^{\sharp}\right\|_{L^{2}\left(Q_{k}\right)}^{2} \\
& -\frac{C}{\delta} \sum_{k=1}^{l^{2}}\left\|\left[\psi_{k}^{\sharp}, H\left(B_{0}\right)\right] u^{\sharp}\right\|_{L^{2}\left(Q_{k}\right)}^{2} \\
= & (1-\delta) q_{11}\left[J_{10} u\right]-\frac{C}{\delta} \sum_{k=1}^{l^{2}}\left\|\left[\psi_{k}^{\sharp}, H\left(B_{0}\right)\right] u^{\sharp}\right\|_{L^{2}\left(Q_{k}\right)}^{2} .
\end{aligned}
$$

For the sum in the R.H.S. of (6.15), we have

$$
\left[\psi_{k}^{\sharp}, H\left(B_{0}\right)\right]=\sum_{j=1}^{2}\left\{2 i\left(\partial_{j} \psi_{k}^{\sharp}\right) \Pi_{j}\left(B_{0}\right)+\left(\partial_{j}^{2} \psi_{k}^{\sharp}\right)\right\} .
$$

Then it follows from (6.10) that

$$
\begin{aligned}
& \sum_{k=1}^{l^{2}}\left\|\left[\psi_{k}^{\sharp}, H\left(B_{0}\right)\right] u^{\sharp}\right\|_{L^{2}\left(Q_{k}\right)}^{2} \\
& \quad \leq \frac{C}{\eta^{2}} \sum_{k=1}^{l^{2}} \sum_{j=1}^{2}\left\|\Pi_{j}\left(B_{0}\right) u^{\sharp}\right\|_{L^{2}\left(Q_{k}\right)}^{2}+\frac{C}{\eta^{4}} \sum_{k}\left\|\chi_{Q_{k}} u^{\sharp}\right\|_{L^{2}\left(Q_{k}\right)}^{2} .
\end{aligned}
$$

For the second sum in the R.H.S. of (6.16), we have

$$
\begin{aligned}
\sum_{k=1}^{l^{2}}\left\|\chi_{Q_{k}} u^{\sharp}\right\|_{L^{2}\left(Q_{k}\right)}^{2} & =\sum_{k=1}^{l^{2}} \int_{Q_{k}}\left|u^{\sharp}(z)\right|^{2} d z \\
& =\sum_{k=1}^{l^{2}} \sum_{n \in Z^{2}} \int_{Q_{k} \cap\left(Q_{R_{2}}+n R_{2}\right)}\left|u^{\sharp}(z)\right|^{2} d z \\
& =\sum_{k=1}^{l^{2}} \sum_{n \in Z^{2}} \int_{\left(Q_{k}-n R_{2}\right) \cap Q_{R_{2}}}\left|u^{\sharp}(z)\right|^{2} d z
\end{aligned}
$$




$$
\begin{aligned}
& =\sum_{k=1}^{l^{2}} \int_{\cup_{n}\left(Q_{k}+n R_{2}\right) \cap Q_{R_{2}}}\left|u^{\sharp}(z)\right|^{2} d z \\
& \leq 9 \int_{Q_{R_{2}}}|u(z)|^{2} d z,
\end{aligned}
$$

where we used the periodicity of $\left|u^{\sharp}(z)\right|$ in the third equality and used (6.12) in the last inequality.

For the first term in the R.H.S. of (6.16), we have, for small $\delta^{\prime}>0$,

$$
\begin{aligned}
& \sum_{j=1}^{2} \sum_{k=1}^{l^{2}}\left\|\Pi_{j}\left(B_{0}\right) u^{\sharp}\right\|_{L^{2}\left(Q_{k}\right)}^{2} \\
& \quad \leq \sum_{j=1}^{2} 9 \int_{Q_{R_{2}}}\left|\Pi_{j}\left(B_{0}\right) u(z)\right|^{2} d z \quad(\text { by }(6.12)) \\
& \quad=9 \sum_{j=1}^{2}\left(\Pi_{j}\left(B_{0}\right)^{2} u, u\right)_{L^{2}\left(Q_{R_{2}}\right)} \quad(\text { by }(6.1)-(6.2)) \\
& \quad \leq \delta^{\prime}\left\|H\left(B_{0}\right) u\right\|_{L^{2}\left(Q_{R_{2}}\right)}^{2}+\frac{C}{\delta^{\prime}}\|u\|_{L^{2}\left(Q_{R_{2}}\right)}^{2} \\
& \quad \leq 2 \delta^{\prime}\left(\left\|\left(H\left(B_{0}\right)+\lambda\right) u\right\|_{L^{2}\left(Q_{R_{2}}\right)}^{2}+\lambda^{2}\|u\|_{L^{2}\left(Q_{R_{2}}\right)}^{2}\right)+\frac{C}{\delta^{\prime}}\|u\|_{L^{2}\left(Q_{R_{2}}\right)}^{2}
\end{aligned}
$$

From (6.16)-(6.18), we have

$$
\begin{aligned}
\sum_{k}\left\|\left[\psi_{k}, H\left(B_{0}\right)\right] u^{\sharp}\right\|^{2} & \\
\leq & C\left(\frac{1}{\eta^{2}}\left(\delta^{\prime}\left\|\left(H\left(B_{0}\right)+\lambda\right) u\right\|_{L^{2}\left(Q_{R_{2}}\right)}^{2}+\left(\frac{1}{\delta^{\prime}}+\delta^{\prime} \lambda^{2}\right)\|u\|_{L^{2}\left(Q_{R_{2}}\right)}^{2}\right)\right. \\
& \left.+\frac{1}{\eta^{4}}\|u\|_{L^{2}\left(Q_{R_{2}}\right)}^{2}\right) .
\end{aligned}
$$

Then it follows from (6.15) and (6.19) that

$q_{10}[u] \geq(1-\delta) q_{11}\left[J_{10} u\right]-\frac{C \delta^{\prime}}{\delta \eta^{2}} q_{10}[u]-C\left(\frac{\delta^{\prime} \lambda^{2}}{\delta \eta^{2}}+\frac{1}{\delta \eta^{2} \delta^{\prime}}+\frac{1}{\delta \eta^{4}}\right)\|u\|_{L^{2}\left(Q_{R_{2}}\right)}^{2}$

Finally, if we set $\delta^{\prime}=\delta^{2} \eta^{2} / C$, we have

$$
\begin{aligned}
q_{10}[u] & \geq \frac{1-\delta}{1+\delta} q_{11}\left[J_{10} u\right]-\frac{C}{1+\delta}\left(\delta \lambda^{2}+\frac{1}{\delta^{3} \eta^{4}}+\frac{1}{\delta \eta^{4}}\right)\|u\|_{L^{2}\left(Q_{R_{2}}\right)}^{2} \\
& \geq(1-2 \delta) q_{11}\left[J_{10} u\right]-C\left(\delta \lambda^{2}+\frac{1}{\delta^{3} \eta^{4}}+\frac{1}{\delta \eta^{4}}\right)\|u\|_{L^{2}\left(Q_{R_{2}}\right)}^{2}
\end{aligned}
$$


for sufficiently small $\delta>0$. This completes the proof.

Applying Lemma 2.1 to the following pair of triplets:

$$
\left(L^{2}\left(Q_{R_{2}}\right), q_{10},\left.\mathcal{E}\left(B_{0}, R_{2}\right)\right|_{Q_{R_{2}}}\right), \quad\left(\bigoplus_{k=1}^{l^{2}} L^{2}\left(Q_{k}\right), q_{11}, \bigoplus_{k=1}^{l^{2}} C_{0}^{\infty}\left(Q_{k}\right)\right)
$$

we have from Lemmas 6.2 and 6.3 , for small $\mu>0$,

$$
N_{q_{10}}\left(\tilde{\mu}_{\lambda \eta \delta}^{2}\right) \leq N_{q_{11}}\left(\mu^{2}\right)
$$

where $\tilde{\mu}_{\lambda \eta \delta}$ is defined by the relation

$$
\tilde{\mu}_{\lambda \eta \delta}^{2}=(1-2 \delta) \mu^{2}-C\left(\delta \lambda^{2}+\frac{1}{\delta^{3} \eta^{4}}+\frac{1}{\delta \eta^{4}}\right)
$$

Then Proposition 6.1 implies that

$$
N_{q_{10}}\left(\tilde{\mu}_{\lambda \eta \delta}^{2}\right)=\frac{B_{0} R_{2}^{2}}{2 \pi} \sharp\left\{k \in N||(2 k-1) B_{0}+\lambda \mid<\tilde{\mu}_{\lambda \eta \delta}\right\}
$$

and

$$
N_{q_{11}}\left(\mu^{2}\right)=l^{2} N_{q}\left(\mu^{2}\right)
$$

where the form $q$ is as in (6.3). From (6.20)-(6.22), it follows that, as $l \rightarrow \infty$,

$$
\begin{aligned}
N_{q}\left(\mu^{2}\right) & \geq \frac{B_{0} R_{2}^{2}}{2 \pi l^{2}} \sharp\left\{k \in N||(2 k-1) B_{0}+\lambda \mid<\tilde{\mu}_{\lambda \eta \delta}\right\} \\
& \geq(l-\varepsilon)^{2} \frac{B_{0}(R-\eta)^{2}}{2 \pi l^{2}} \sharp\left\{k \in N||(2 k-1) B_{0}+\lambda \mid<\tilde{\mu}_{\lambda \eta \delta}\right\},
\end{aligned}
$$

where we used the relation (6.8) in the last inequality.

Now we complete the proof of Proposition 2.1 (ii), since

$$
\delta \lambda^{2}+\frac{1}{\delta^{3} \eta^{4}}+\frac{1}{\delta \eta^{4}} \leq C\left(\delta \lambda^{2}+\frac{1}{\delta^{3} \eta^{4}}\right)
$$

holds for $\delta>0$ sufficiently small and hence $\mu_{\lambda \eta \delta} \leq \tilde{\mu}_{\lambda \eta \delta}$. 


\section{References}

[A-H-S] Avron, J., Herbst, I. and Simon, B., Schrödinger operators with magnetic fields I. General interactions, Duke Math. J., 45(4) (1978), 847-883.

[Col] Colin de Verdiere, Yves., L'asymptotique de Weyl pour les bouteilles magnétiques, Comm. Math. Phys., 105 (1986), 327-335.

[E-K] Exner, P. and Kovarik, H., Magnetic strip waveguides, J. Phys. A., 33 (16) (2000), 3297-3311.

[Iwa] Iwatsuka, A., Examples of absolutely continuous Schrödinger operators in magnetic fields, Publ. RIMS, Kyoto Univ., 21 (1985), 385-401.

[Kat] Kato, T., Perturbation Theory for Linear Operator, Springer, Berlin-HeidelbergNew York, 1976.

[L-S] Leinfelder, H. and Simader, C. G., Schrödinger operators with singular magnetic potentials, Math. Z., 176 (1981), 1-19.

[M-P] Mântoiu, M. and Purice, R., Some propagation properties of the Iwatsuka model, Comm. Math. Phys., 188 (1997), 691-708.

[Rai1] Raikov, G. D., Eigenvalue asymptotics for the Schrödinger operator with homogeneous magnetic potential and decreasing electric potential I. Behaviour near the essential spectrum tips, Comm. in P.D.E., 15(3) (1990), 407-434.

[Rai2] - Border-line eigenvalue asymptotics for the Schrödinger operator with electromagnetic potential, Integral Eq. and Operator Theory, 14 (1991), 875-888.

[R-S1] Reed, M. and Simon, B., Methods of Modern Mathematical Physics, Vol. I, Academic Press, New York, 1972.

[R-S2] - Methods of Modern Mathematical Physics, Vol. II, Academic Press, New York, 1975.

[R-S4] ․ Methods of Modern Mathematical Physics, Vol. IV, Academic Press, New York, 1978.

[Tam] Tamura, H., Asymptotic distribution of eigenvalues for Schrödinger operators with homogeneous magnetic fields, Osaka J. Math., 25 (1988), 633-647. 